Non-linear stress response of non-gap-spanning magnetic chains suspended in a Newtonian fluid under oscillatory shear test: A direct numerical simulation

M. R. Hashemi, M. T. Manzari, and R. Fatehi

Citation: Physics of Fluids 29, 107106 (2017);

View online: https://doi.org/10.1063/1.5009360

View Table of Contents: http://aip.scitation.org/toc/phf/29/10

Published by the American Institute of Physics

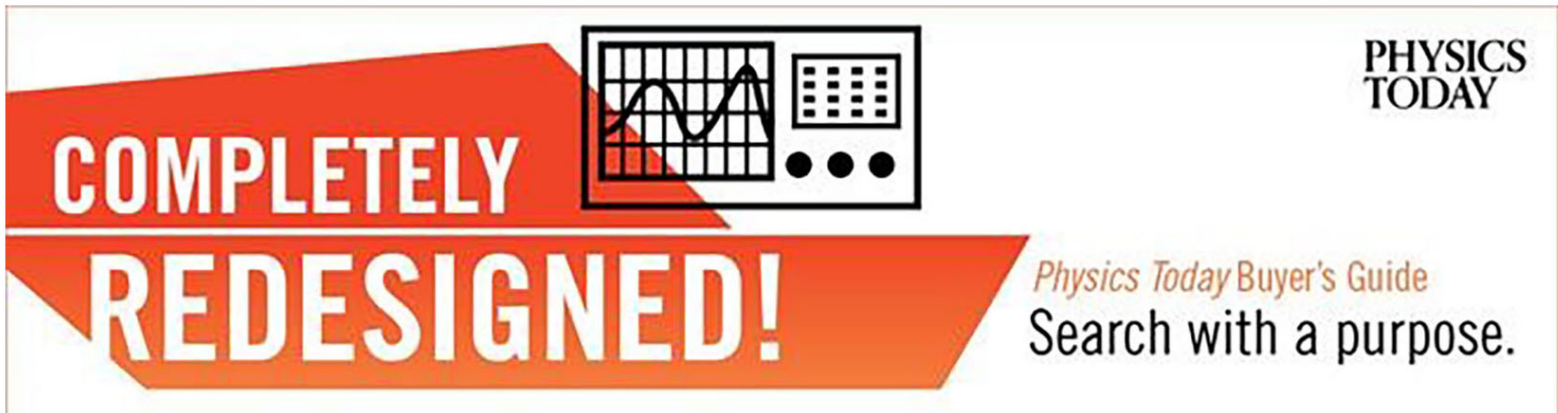




\title{
Non-linear stress response of non-gap-spanning magnetic chains suspended in a Newtonian fluid under oscillatory shear test: A direct numerical simulation
}

\author{
M. R. Hashemi, ${ }^{1}$ M. T. Manzari, ${ }^{1,2, a)}$ and R. Fatehi ${ }^{3,4}$ \\ ${ }^{1}$ Center of Excellence in Energy Conversion, School of Mechanical Engineering, Sharif University of Technology, \\ Tehran, Iran \\ ${ }^{2}$ School of Geosciences, University of Aberdeen, Aberdeen, United Kingdom \\ ${ }^{3}$ Department of Mechanical Engineering, Persian Gulf University, Bushehr 75168, Iran \\ ${ }^{4}$ Oil and Gas Research Center, Persian Gulf University, Bushehr 75169, Iran
}

(Received 25 May 2017; accepted 15 October 2017; published online 31 October 2017)

\begin{abstract}
A direct numerical simulation approach is used to investigate the effective non-linear viscoelastic stress response of non-gap-spanning magnetic chains suspended in a Newtonian fluid. The suspension is confined in a channel and the suspended clusters are formed under the influence of a constant external magnetic field. Large amplitude oscillatory shear (LAOS) tests are conducted to study the non-linear rheology of the system. The effect of inertia on the intensity of non-linearities is discussed for both magnetic and non-magnetic cases. By conducting magnetic sweep tests, the intensity and quality of the non-linear stress response are studied as a function of the strength of the external magnetic field. The Chebyshev expansion of the stress response is used to quantify the non-linear intra-cycle behaviour of the suspension. It is demonstrated that the system shows a strain-softening behaviour while the variation of the dynamic viscosity is highly sensitive to the external magnetic field. In a series of strain sweep tests, the overall non-linear viscoelastic behaviour of the system is also investigated for both a constant frequency and a constant strain-rate amplitude. It is shown that the intra-cycle behaviour of the system is different from its inter-cycle behaviour under LAOS tests. Published by AIP Publishing. https://doi.org/10.1063/1.5009360
\end{abstract}

\section{INTRODUCTION}

The bulk rheology of electrorheological (ER) and magnetorheological (MR) fluids can be readily adjusted by applying an external electric and magnetic field, respectively. ${ }^{1,2}$ This makes these fluids suitable choices for active control mechanisms, e.g., dampers and actuators. ${ }^{3-5}$ Under the influence of an external field, a micro-structure is formed by particle aggregates aligned with the direction of the field. This microstructure can lead to either a significant viscosity enhancement or a solid-like behaviour depending on the strength of the induced bonds and the concentration of the solid particles. ${ }^{6}$ Generally, magnetic bonds are stronger in a conventional magnetorheological (MR) fluid than electric bonds in an electrorheological (ER) fluid $;^{7}$ therefore, MR fluids have become more attractive in recent years.

Under a steady shear test, as long as the static yield stress $^{8}$ of an MR fluid is not exceeded, there will not be any flow. Above this static yield stress threshold, the static frictional force exerted by ending particles in the micro-structure is overcome ${ }^{9}$ and an infinite strain is possible. ${ }^{10}$ By further increasing the shear strain, a strain-softening behaviour is observed due to breakdown of the magnetic clusters. When a field-induced (chain-like) structure is strained to a rather large extent, it becomes unstable and eventually breaks apart. ${ }^{11}$ At this point, the MR fluid flows with a finite strain-rate and the

a)E-mail: mtmanzari@sharif.edu associated stress is the so-called dynamic (or Bingham ${ }^{10}$ ) yield stress. ${ }^{12}$ Both the static and dynamic yield stresses are functions of intensity of the magnetic field, particle concentration, and particle size distribution. ${ }^{6,13,14}$ In the post-yield state, the behaviour of MR fluids is generally shear-thinning. ${ }^{15,16}$

Under an oscillatory shear test, MR fluids exhibit a viscoelastic behaviour with moduli that primarily depend on their micro-structure. ${ }^{17}$ The linear viscoelastic behaviour of MR fluids has been thoroughly investigated especially for the pre-yield state. ${ }^{18,19}$ Nevertheless, MR fluids exhibit a linear behaviour only in a very narrow range of strain amplitude. ${ }^{8,20}$ Large Amplitude Oscillatory Shear (LAOS) tests can be utilized to investigate the nonlinear rheological behaviour of MR fluids. The LAOS test reveals that MR fluids can be classified as type III (complex fluids) which exhibits a strain-softening/shear-thinning behaviour with a slight overshoot in the loss modulus. ${ }^{8,21}$ The nonlinear behaviour of field responsive (ER and MR) fluids is commonly attributed to the breakdown ${ }^{20,22}$ and rearrangement ${ }^{23}$ of the particle clusters.

As discussed in Ref. 24, an MR fluid behaves as an elasto-visco-plastic material ${ }^{25}$ whose micro-structure has the principal role in determining its bulk rheology. This role can be explored using a particle-level numerical simulation. ${ }^{26,27}$ In the literature, numerical simulation has been widely employed to investigate various aspects of the MR fluids, e.g., time scales associated with magnetic chain formation, ${ }^{28}$ particle aggregation in a poly-disperse magnetic suspension, ${ }^{12}$ micro-structural evolution in a Poiseuille flow, ${ }^{29}$ and magnetic 
clusters exposed to an oscillatory shear test. ${ }^{19}$ Theoretical models ${ }^{7,30,31}$ are also useful for evaluating the storage modulus in the linear region ${ }^{17,19}$ and estimating the dynamic yield stress. ${ }^{16}$

In the majority of previous particle-level simulations and theoretical models addressing the effective rheology of a fieldresponsive fluid, the field-induced chains were considered to be gap-spanning with ending particles stuck to the channel walls. ${ }^{19,32}$ For a rather large strain amplitude, these gapspanning clusters undergo progressive rearrangements ${ }^{33}$ and eventually break up into smaller non-gap-spanning chains by further increase in the strain amplitude. Since a magnetic chain would most probably break from its tip, ${ }^{34}$ the blockage ratio associated with the shortened clusters is still large enough to significantly affect rheology of the system. However, the individual contribution of these clusters to the bulk rheology has been rarely addressed in the literature. A successful modeling of these broken non-gap-spanning magnetic chains needs a two-way coupling between the suspending fluid flow and the suspended solid particles, which necessitates utilization of a direct numerical simulation (DNS) approach. ${ }^{35}$

Recently, using the DNS approach, it has been shown that non-gap-spanning chains can also contribute to the storage of energy $^{36}$ as well as enhance the effective viscosity. ${ }^{37,38}$ In that work ${ }^{36}$ a confined periodic array of non-gap-spanning magnetic chains was suspended in a Newtonian fluid exposed to a small amplitude oscillatory shear (SAOS). It was shown that the system behaves as a viscoelastic fluid. It was also discussed how inertia could hinder elasticity, an effect which can be controlled by adjusting the intensity of the external magnetic field. The main goal of the present work is to qualitatively investigate the non-linear stress response of the non-gap-spanning magnetic clusters. To this end, an array of suspended magnetic clusters similar to the systems presented in the previous studies $^{36,37}$ is simulated under LAOS and the effective stress response is studied following the methodology introduced in the literature. ${ }^{39-41}$ In the following, first the physical model and the governing equations are briefly described. Then, the results of the LAOS tests are presented and the non-linearities in the intra-cycle and overall rheology of the system are discussed. The methodologies used to characterize the results of the LAOS tests are also briefly surveyed during discussions.

\section{PHYSICAL MODELING}

A direct numerical simulation (DNS) approach is used to investigate the behaviour of a suspension of paramagnetic solid particles forming non-gap-spanning chain-like clusters. Here, the physical system consists of a Newtonian fluid and a number of (para-)magnetic solid particles confined between two parallel walls. The system is subjected to a large amplitude oscillatory shear (LAOS) test as schematically shown in Fig. 1(a). In order to avoid a prohibitive computational cost, the study is performed on a two-dimensional periodic domain as shown in Fig. 1(b). The computational domain contains $N$ neutrally buoyant circular cylinders initially arranged in a vertical row with the middle one being placed at the center. These solid particles are magnetized under the influence of an external magnetic field with a flux density of $B_{0}$. For the current setup, in order to study the shear rheology of the system, the spatially averaged stress response is measured as

$$
\bar{\sigma}_{x y}=\frac{1}{L} \int_{y=0} \sigma_{x y}(x) d x,
$$

where $\sigma_{x y}$ is the local value of the shear stress. In the following, the over-bar sign is omitted for brevity.

In the present work, the smoothed particle hydrodynamics (SPH) method ${ }^{38}$ is used to solve the governing equations for both the fluid flow and the magnetostatics. For fluid flows, in the Lagrangian framework of the weakly compressible SPH method, ${ }^{42}$ the governing equations are the conservation of momentum

and the continuity

$$
\rho \frac{d \mathbf{v}}{d t}=-\nabla p+\eta_{0} \nabla^{2} \mathbf{v}
$$

$$
\frac{d \rho}{d t}=-\rho \nabla \cdot \mathbf{v},
$$

where a simple equation of state, $p-p_{0}=c_{0}^{2}\left(\rho-\rho_{0}\right)$, relates density and pressure. Here, the velocity vector $\mathbf{v}$ is subject to the no-slip boundary condition at a solid surface. In these equations, $\rho$ is the density, $p$ is the pressure, and $\eta_{0}$ denotes the dynamic viscosity of the suspending fluid. Also, $c_{0}$ is the artificial speed of sound and subscript 0 denotes the initial state in the fluid domain.

For a two-dimensional magnetic field, in the absence of a free current, the Maxwell equations ${ }^{43}$ can be combined into the Poisson equation for the magnetic potential, $\phi$, as

$$
\nabla \cdot(\mu \nabla \phi)=0 .
$$

In this way, the magnetic field intensity is calculated as $\mathbf{H}=\nabla \phi$. Far below the magnetic saturation limit, ${ }^{44}$ constant magnetic permeabilities are considered for the solid bodies $\left(\mu_{s}\right)$ and the fluid domain $\left(\mu_{0}\right)$. The magnetic flux density is calculated as

$$
\mathbf{B}=\mu \mathbf{H} .
$$

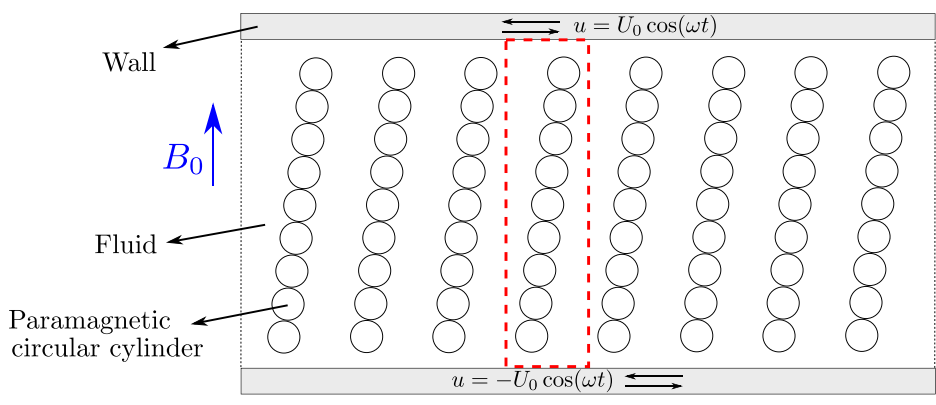

(a)

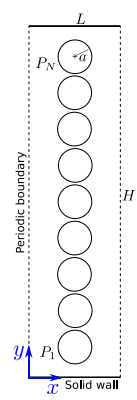

(b)
FIG. 1. Schematic of (a) the suspension of magnetic solid particles shearing in a channel with oscillating solid walls and (b) the initial configuration of the particles in the computational domain. The computational domain is marked by dashed lines in (a). 
The magnetic field is subject to the conservation of $\mathbf{B}$ at the fluid-solid interface. It must be noted that the external magnetic field is imposed by setting $\phi$ at the solid walls so that $\mathbf{B}_{0}=\mu_{0} \nabla \phi$ as explained in the literature. ${ }^{35,38,45}$

Solid bodies are moved using Newton's law of motion as

$$
M_{s} \frac{d \mathbf{v}_{s}}{d t}=\mathbf{F}_{s}^{m}+\mathbf{F}_{s}^{h}+\mathbf{F}_{s}^{r}
$$

and

$$
I_{s} \frac{d \mathbf{\Omega}_{s}}{d t}=\mathbf{M}_{s}^{m}+\mathbf{M}_{s}^{h},
$$

where $M_{s}$ and $I_{s}$ are the total mass and moment of inertia of solid body $s$, respectively. Also, $\mathbf{v}_{s}$ and $\boldsymbol{\Omega}_{s}$ are the linear and angular velocities of $s$, respectively. The terms on the right-hand side of Eq. (6) are the magnetic force, $\mathbf{F}^{m}$, the hydrodynamic force, $\mathbf{F}^{h}$, and the repulsive force due to solid-solid collisions, $\mathbf{F}^{r}$. In a similar way, the terms on the right-hand side of Eq. (7) correspond to the magnetic and hydrodynamic effects. The full description of the numerical method and boundary conditions was presented in an earlier article. $^{38}$

\section{A. Simulation details}

In all test cases solved in this paper, circular cylinders are of the same radius $(a)$; the number of solid particles initially arranged in a chain, $N=9$, the non-dimensionalized channel height, $H / a=20$, and the non-dimensionalized periodicity length, $L / a=8$, are kept constant. This gives a solid volume fraction of $N \pi a^{2} / L H \approx 0.177$. In order to facilitate the numerical simulation, solid bodies are initially arranged with a vertical spacing equal to the discretization length, $\delta_{p}$. Here, using a rather small ratio for the magnetic permeabilities $\left(\mu_{s} / \mu_{0}=1.1\right)$, converged solutions are obtained for $a / \delta_{p}=18.75$. As discussed previously, when exposed to an external magnetic field, this system exhibits a viscoelastic behaviour. The shear rheology of such a system can be investigated using an oscillatory shear test. This study aims to extend the results obtained in the previous work ${ }^{36}$ to LAOS.

In this work, inertia is quantified at the particle scale by defining the particle Reynolds number as $R e_{p}=\rho \dot{\gamma}_{0} a^{2} / \eta_{0}$. Also, as discussed in the literature, ${ }^{15,46}$ for a steady shear flow, the viscous force can be non-dimensionalized against the magnetic force using the Mason number defined as

$$
M n=\frac{\dot{\gamma}_{0} \eta_{0}}{\mu_{0} \beta^{2} H_{0}^{2}},
$$

where $\dot{\gamma}_{0}=2 U_{0} / H, \beta=\chi /(3+\chi)$ is the effective polarization, and $\chi=\left(\mu_{s}-\mu_{0}\right) / \mu_{0}$ is the magnetic susceptivity. On the other hand, for an oscillatory shear test, since the time scale can be properly determined by the frequency of oscillations $(\omega)$, a modified non-dimensional group is defined and used in the present work,

$$
M n^{*}=\operatorname{Mn} \frac{\omega}{\dot{\gamma}_{0}} .
$$

It must be noted that in Sec. III B, since $H_{0}$ is the variable and $\omega$ is constant, $\left(M n^{*}\right)^{-1}$ is used as a measure of the external magnetic field. Also, in Sec. III C, where $\omega$ is the variable and $\dot{\gamma}_{0}$ is constant, $M n$ as defined in Eq. (8) is used as a measure of the external magnetic field.

\section{B. LAOS theory}

The stress response of a viscoelastic material to an oscillatory shear strain, $\gamma(t)=\gamma_{0} \sin (\omega t)$, is harmonic with the same frequency, $\omega$, only for a rather small strain amplitude. In a more general representation which is also valid for a LAOS test, the stress response can be described using the Fourier series as ${ }^{47,48}$

$$
\sigma_{x y}=\gamma_{0} \sum_{n: o d d}\left|G_{\mathrm{n}}^{*}\right|\left(\omega, \gamma_{0}\right) \sin \left(n \omega t+\Psi_{\mathrm{n}}\right) .
$$

Here, $G_{\mathrm{n}}^{*}$ and $\Psi_{\mathrm{n}}$ are the complex modulus and phase angle corresponding to the $n$th harmonic, respectively. For a SAOS test, only the first harmonic is important, while for a LAOS test, higher harmonics are also significant. In this work, using the subroutines provided in the MITlaos ${ }^{40,49}$ program, the stress response is calculated using only the first, third, and fifth harmonics. Normally, amplitudes of the higher harmonics are either negligible or an order of magnitude smaller than the third harmonic. It should be noted that for an odd-symmetric stress response, even harmonics are all negligible. ${ }^{50}$

The stress response can be decomposed using its symmetry properties, ${ }^{40}$ and considering the fact that elasticity and viscosity are related to the storage and loss of energy, respectively, the elastic stress, $\sigma^{\prime}$, and the viscous stress, $\sigma^{\prime \prime}$, are obtained as ${ }^{50}$

$$
\sigma_{x y}^{\prime}=\frac{\sigma_{x y}(\gamma, \dot{\gamma})-\sigma_{x y}(-\gamma, \dot{\gamma})}{2}
$$

and

$$
\sigma_{x y}^{\prime \prime}=\frac{\sigma_{x y}(\gamma, \dot{\gamma})-\sigma_{x y}(\gamma,-\dot{\gamma})}{2} .
$$

In this way, $d \sigma^{\prime} / d \gamma$ and $d \sigma^{\prime \prime} / d \dot{\gamma}$ are measures of the local (tangent) elastic modulus and dynamic viscosity, respectively.

In order to quantify the non-linear properties of the (intracycle) rheology of the system, it is more appropriate to express the elastic (and viscous) stress as a polynomial series ${ }^{40,50}$ rather than using the above mentioned Fourier Transform (FT) rheology. ${ }^{39}$ To this end, a framework has been introduced by Ewoldt et al. ${ }^{48}$ that facilitates the physical interpretation of the non-linear rheology of a material under LAOS. ${ }^{51}$ The idea is to expand $\sigma_{x y}^{\prime}$ and $\sigma_{x y}^{\prime \prime}$ in series of the Chebyshev polynomials of the first kind, $\mathrm{T}_{\mathrm{n}}$, as ${ }^{48}$

$$
\sigma_{\mathrm{xy}}^{\prime}(\gamma)=\gamma_{0} \sum_{n: o d d} e_{\mathrm{n}}\left(\omega, \gamma_{0}\right) \mathrm{T}_{\mathrm{n}}\left(\frac{\gamma}{\gamma_{0}}\right)
$$

and

$$
\sigma_{\mathrm{xy}}^{\prime \prime}(\dot{\gamma})=\dot{\gamma}_{0} \sum_{n: \text { odd }} v_{\mathrm{n}}\left(\omega, \gamma_{0}\right) \mathrm{T}_{\mathrm{n}}\left(\frac{\dot{\gamma}}{\dot{\gamma}_{0}}\right),
$$

where $e_{\mathrm{n}}=G_{\mathrm{n}}^{\prime}(-1)^{(n-1) / 2}$ and $v_{\mathrm{n}}=\eta_{\mathrm{n}}^{\prime}$. Considering only the first and third harmonics in the stress response, the resulting polynomials are

$$
\sigma_{\mathrm{xy}}^{\prime}(\gamma) \approx\left(e_{1}-3 e_{3}\right) \gamma+4 e_{3} \frac{\gamma^{3}}{\gamma_{0}^{2}}
$$

and

$$
\sigma_{\mathrm{xy}}^{\prime \prime}(\dot{\gamma}) \approx\left(v_{1}-3 v_{3}\right) \dot{\gamma}+4 v_{3} \frac{\dot{\gamma}^{3}}{\dot{\gamma}_{0}^{2}}
$$


According to Eqs. (15) and (16), $e_{3}$ and $v_{3}$ determine the variation of the tangent elastic modulus $\left(d \sigma^{\prime} / d \gamma\right)$ and the tangent dynamic viscosity $\left(d \sigma^{\prime \prime} / d \dot{\gamma}\right)$ in a strain-cycle; a positive $e_{3}$ leads to an intra-cycle strain-stiffening behaviour and an intracycle shear-thickening behaviour is associated with a positive $v_{3}$. Negative $e_{3}$ and $v_{3}$ also correspond to the intra-cycle strain-softening and shear-thinning behaviours, respectively.

\section{RESULTS}

In the following, first, the effect of inertia on the non-linear rheology of the system is investigated for both non-magnetic and paramagnetic solid particles. In Secs. III B and III C, the results of magnetic sweep tests (changing the intensity of the external magnetic field while keeping all other parameters constant) and strain sweep tests are presented, respectively.

\section{A. Non-linearity and the effects of inertia}

It is easy to show that for a linear stress response, the Lissajous-Bowditch curve, ${ }^{52}$ a plot of stress versus strain (-rate), is of an elliptical shape. By increasing the strain amplitude in a LAOS test, higher harmonics become more significant and non-elliptical Lissajous-Bowditch curves are obtained.

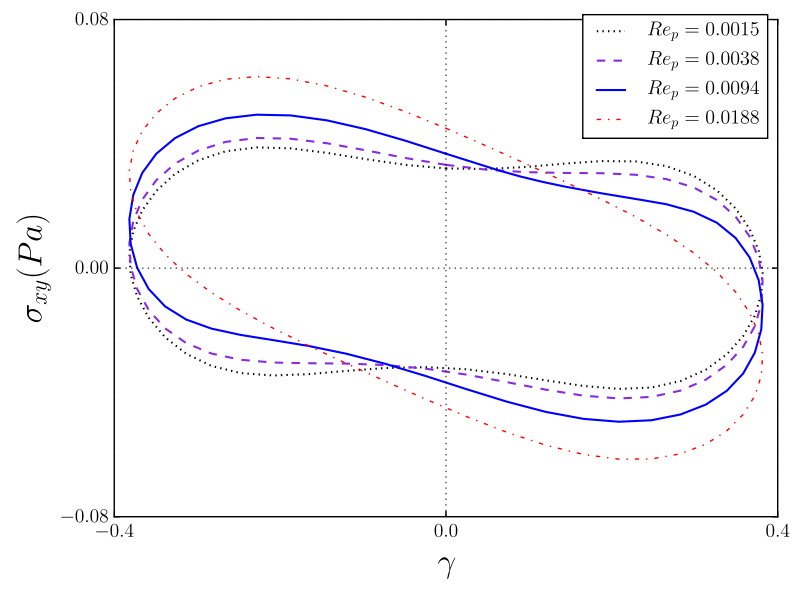

(a)

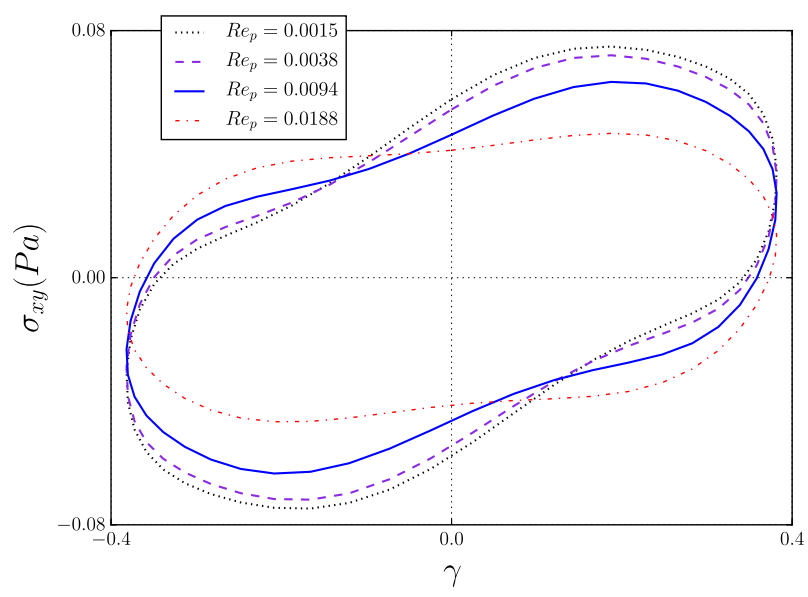

(c)
These curves are helpful for a qualitative interpretation of the results by investigating the variation of the stress response in a complete strain(-rate) cycle. ${ }^{50,52}$ In Fig. 2, Lissajous-Bowditch curves are shown for $\gamma_{0}=1.2 / \pi$ with $\omega=2 \pi \mathrm{rad} / \mathrm{s}$ and different particle Reynolds numbers. Here, $0.0015 \leq R e_{p} \leq 0.0188$ is changed by altering density while all other parameters are kept constant. Results are presented for both the non-magnetic case and the magnetic case with $M n^{*}=0.419$.

Previously, ${ }^{36}$ it was discussed that for a purely viscous system (or a viscoelastic system with weak elasticity) with finite inertia, an obtuse phase angle, i.e., $\Psi_{1}>\pi / 2$, is obtained. The larger the Reynolds number, the larger the phase angle is. The effect of inertia on the orientation of the LissajousBowditch curve is schematically shown in Fig. 3(a). For the present test cases in the absence of an external magnetic field, the stress response is almost purely viscous and a similar behaviour is observed in Fig. 2(a). Also for a viscoelastic system, by increasing inertia, the phase angle increases and consequently the effective elasticity $\left(G^{\prime}\right)$ is decreased ${ }^{36}$ and the Lissajous-Bowditch curve rotates in the clockwise direction as schematically shown in Fig. 3(b). For the present test cases with $M n^{*}=0.419$, the stress response is viscoelastic and a similar behaviour is observed in Fig. 2(c). It is also worth

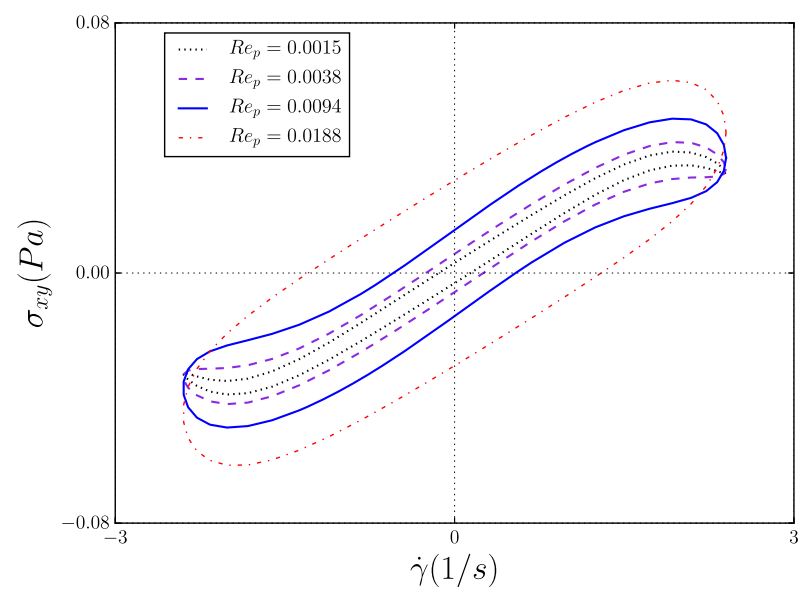

(b)

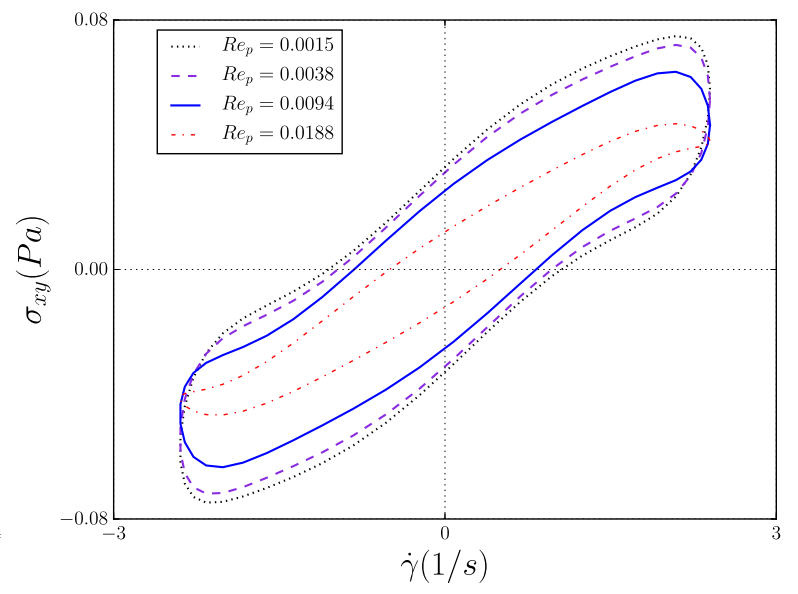

(d)

FIG. 2. Lissajous-Bowditch curves obtained for $\dot{\gamma}_{0}=2.4 \mathrm{~s}^{-1}, \omega=2 \pi \mathrm{rad} / \mathrm{s}$, and different Reynolds numbers, with $B_{0}=0$ for (a) and (b) and $M n^{*}=0.419$ for (c) and (d). 


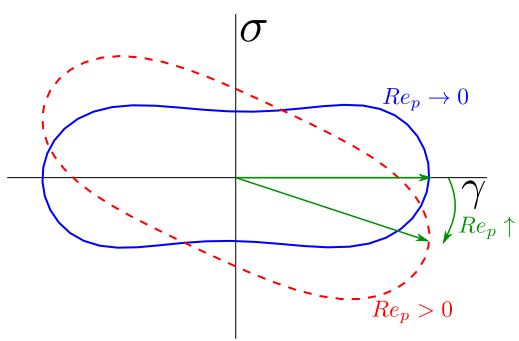

(a)

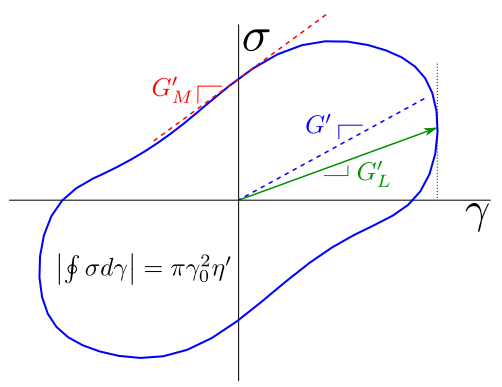

(c)

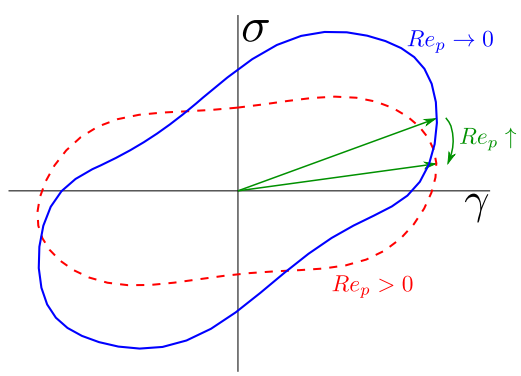

(b)

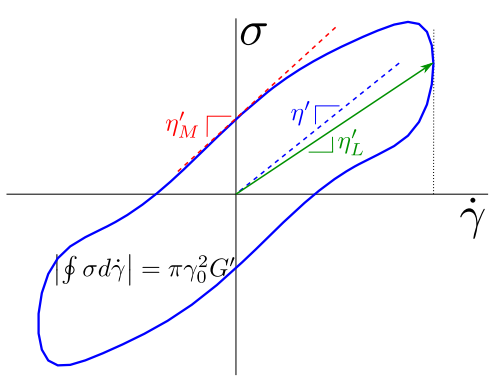

(d)
FIG. 3. Schematic representation of the LissajousBowditch curves showing the effect of inertia for purely viscous (a) and viscoelastic (b) cases and the graphical definition of elastic moduli (c) and dynamic viscosities (d). noting that for the non-magnetic case, by increasing inertia, the shape of the Lissajous-Bowditch curves becomes more like an ellipse. This is a sign of reduction in the intensity of the non-linearities.

Also using the Lissajous-Bowditch curves, the variation of $\sigma_{\mathrm{xy}}$ in a complete LAOS strain(-rate) cycle can be studied and the non-linear response of a system can be understood. ${ }^{40}$ The geometrical representation of the elastic modulus at zero strain, $G_{M}^{\prime}=\left.(d \sigma / d \gamma)\right|_{\gamma=0}$, the elastic modulus at maximum strain, $G_{L}^{\prime}=\left.(\sigma / \gamma)\right|_{\gamma=\gamma_{0}}$, the dynamic viscosity at zero strain-rate, $\eta_{M}^{\prime}=\left.(d \sigma / d \dot{\gamma})\right|_{\dot{\gamma}=0}$, and the dynamic viscosity at maximum strain-rate, $\eta_{L}^{\prime}=\left.(\sigma / \dot{\gamma})\right|_{\dot{\gamma}=\dot{\gamma}_{0}}$, are shown in Figs. 3(c) and 3(d). The value of average elastic modulus, $G^{\prime}$, is always between $G_{M}^{\prime}$ and $G_{L}^{\prime}$ and can be calculated from the area enclosed by the viscous Lissajous-Bowditch curve (plot of $\sigma$ versus $\dot{\gamma}$ ). A similar statement is also valid for $\eta^{\prime}, \eta_{M}^{\prime}$, and $\eta_{L}^{\prime}{ }^{50,53}$ As shown in Fig. 2(c) for $M n^{*}=0.419, G_{L}^{\prime}$ is smaller than $G_{M}^{\prime}$ and therefore the non-linear viscoelastic stress response exhibits a strainsoftening behaviour in a LAOS cycle. Also in a LAOS cycle, the stress response appears to be shear-thinning $\left(\eta_{L}^{\prime}<\eta_{M}^{\prime}\right)$ for both the non-magnetic case and the magnetic case with $M n^{*}=0.419$ as observed in Figs. 2(b) and 2(d). As seen in Fig. 2(b), the area enclosed by $\sigma_{\mathrm{xy}}-\dot{\gamma}$, which is a measure of $\left|G^{\prime}\right|$, increases by increasing $R e_{p}$, while this trend is reversed for $M n^{*}=0.419$. As shown in the previous work, ${ }^{36}$ the reason is that for a non-magnetic case (or cases with a weak magnetic field intensity in which inertial effects are dominant and $\Psi_{1}>\pi / 2$ ), the measured value of $\left|G^{\prime}\right|$ is an increasing function of $R e_{p}$. On the other hand, for cases in which magnetic forces dominate inertia $\left(\Psi_{1}<\pi / 2\right),\left|G^{\prime}\right|$ is a decreasing function of the Reynolds number. Here, this is seen for $M n^{*}=0.419$.

Generally in a LAOS test, the intensity of the third harmonic normalized with the amplitude of the first harmonic, $\left|G_{3}^{*}\right| /\left|G_{1}^{*}\right|$, can be considered as a measure of the nonlinearity of the stress response. Figure 4 presents the variation of $\left|G_{3}^{*}\right| /\left|G_{1}^{*}\right|$ as a function of $R e_{p}$ for the cases shown in Fig. 2 . The ratio $\left|G_{3}^{*}\right| /\left|G_{1}^{*}\right|$ significantly decreases by increasing $R e_{p}$ for a non-magnetic suspension. However, the intensity of nonlinearities is only affected weakly by inertia for a finite external magnetic field with $M n^{*}=0.419$. The arrangement of the solid particles is shown in Fig. 5, which corresponds to the instance that solid particle $P_{1}$ (bottom tip particle) has reached its maximum horizontal displacement. This is equivalent to the maximum (tilting) deflection of the chain of solid particles in a cycle. For the non-magnetic case, it is observed that the particle chain loses its symmetric shape (centered in the channel) for $R e_{p}=0.0038$, while with $M n^{*}=0.419$, the symmetry is retained for all Reynolds numbers. Such an asymmetry can be initiated by any minute asymmetry in the discretization of the computational domain. In practice, any unsymmetrical

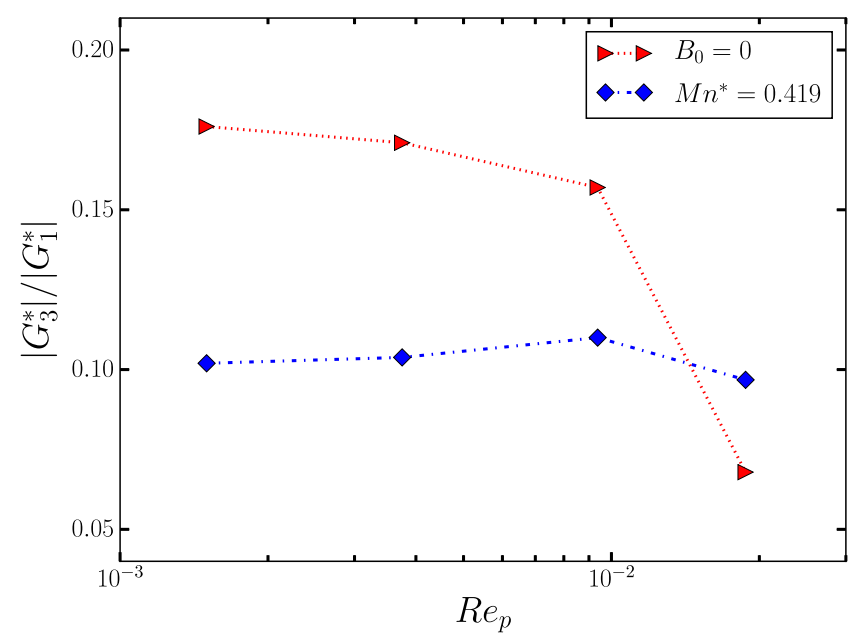

FIG. 4. $\left|G_{3}^{*}\right| /\left|G_{1}^{*}\right|$ as a function of $R e_{p}$ for $\dot{\gamma}_{0}=2.4 \mathrm{~s}^{-1}$ and $\omega=2 \pi \mathrm{rad} / \mathrm{s}$. 

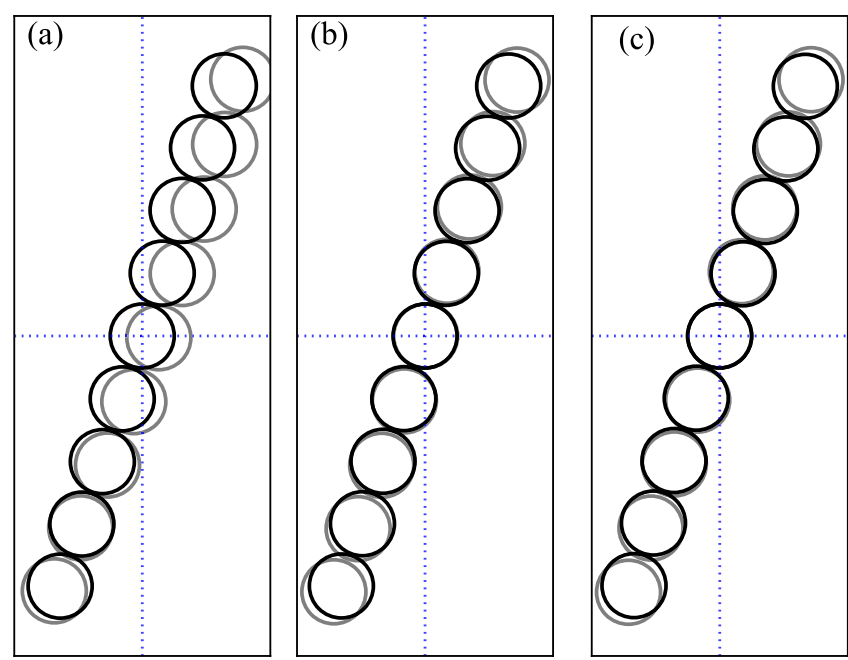

FIG. 5. Position of solid particles obtained for $\gamma_{0}=1.2 / \pi$ and $\omega=2 \pi \mathrm{rad} / \mathrm{s}$. Results obtained for the non-magnetic cases are shown with light color while dark circles depict the cases with $M n^{*}=0.419$. (a) $R e_{p}=0.0038$; light: $\omega t=2.52 \pi$, dark: $\omega t=2.38 \pi$. (b) $R e_{p}=0.0094$; light: $\omega t=2.52 \pi$, dark: $\omega t=2.40 \pi$. (c) $R e_{p}=0.0188$; light: $\omega t=2.56 \pi$, dark: $\omega t=2.44 \pi$.

force can initiate, support, and magnify such an asymmetry. However, for $M n^{*}=0.419$, magnetic bonding forces dominate the hydrodynamic interaction between solid particles and even for $R e_{p}=0.0038$, the initial symmetry is almost undisturbed.

In Fig. 6, time histories of the spatial displacement of $P_{1}$ and $P_{5}$ (the middle solid particle in the chain) are shown for the non-magnetic case with $R e_{p}=0.0038$ and 0.0188 . In the non-magnetic case, solid particles are subject to the hydrodynamic interaction ${ }^{54}$ that opposes the separation of solid particles while the chain is strained. In this case, the net hydrodynamic force is attractive. On the other hand, when particles are forced to return to the vertical arrangement, the net hydrodynamic force is repulsive. This leads to a periodic vertical motion of the solid particle as seen in Fig. 6(b) for $P_{1}$. As long as the micro-structure retains its vertical symmetry, the net force is (almost) zero for $P_{5}$. However, once $P_{5}$ moves away from the centerline, it is subject to non-zero horizontal and vertical forces. There is also another mechanism which works to bring $P_{5}$ back to the centerline; at a finite Reynolds number, a lift force acts on a solid body suspended in a (Newtonian) shear flow. ${ }^{55}$ For $R e_{p}=0.0038$, the lift force is not strong enough to readily compensate for the vertical force exerted on $P_{5}$ due to unbalanced lubrication forces. As a result of the superposition of all active forces, a periodic vertical motion is observed. For higher particle Reynolds numbers, once $P_{5}$ is displaced, the lift force is large enough to promptly bring it back to the centerline. In this work, the results are almost qualitatively similar for $R e_{p}=0.0094$ and 0.0038 . Therefore, in order to avoid prohibitive computational costs, in the rest of this paper, the particle Reynolds number is set to $R e_{p}=0.0094$.

Before further studying the stress response in magnetic sweep tests, it is worth investigating the effect of the number of solid particles arranged in a magnetic cluster on the intensity of the non-linearity; the larger the number of magnetic particles, the larger is the blockage ratio $(2 a N / H)$ in the test channel. It is evident that a larger blockage ratio leads to an increase in the intensity of the measured stress response. Also, if inertia was negligible, by decreasing the number of solid particles that form the magnetic chain, $\left|G_{1}^{*}\right| / \eta_{0} \omega$ tends to unity. However, the curve shown in Fig. 7(a) approaches a larger than unity value at the limit of $N \rightarrow 0$ due to a finite Reynolds number. Inertia increases the rate of energy dissipation in an oscillatory shear test ${ }^{56}$ and, in this sense, adds to the amplitude of the measured stress. Moreover, it is expected that the intensity of the nonlinearity of the stress response decreases by shortening the magnetic cluster (or reducing the solid volume fraction). This is observed in Fig. 7(b) where $\left|G_{3}^{*}\right| /\left|G_{1}^{*}\right|$ tends to zero as $N \rightarrow 0$.

\section{B. Magnetic sweep tests}

In the so called magnetic sweep tests, all parameters are kept constant, while the intensity of the external magnetic field

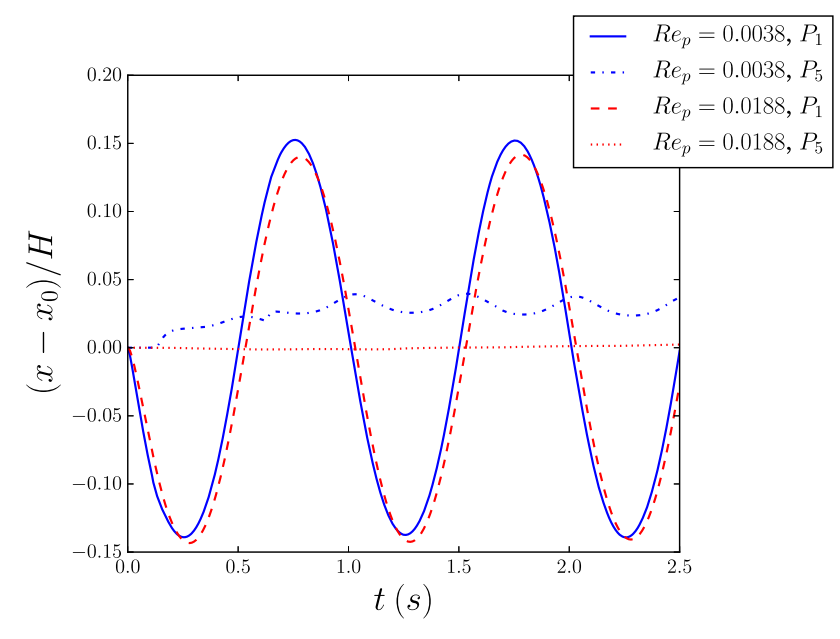

(a)

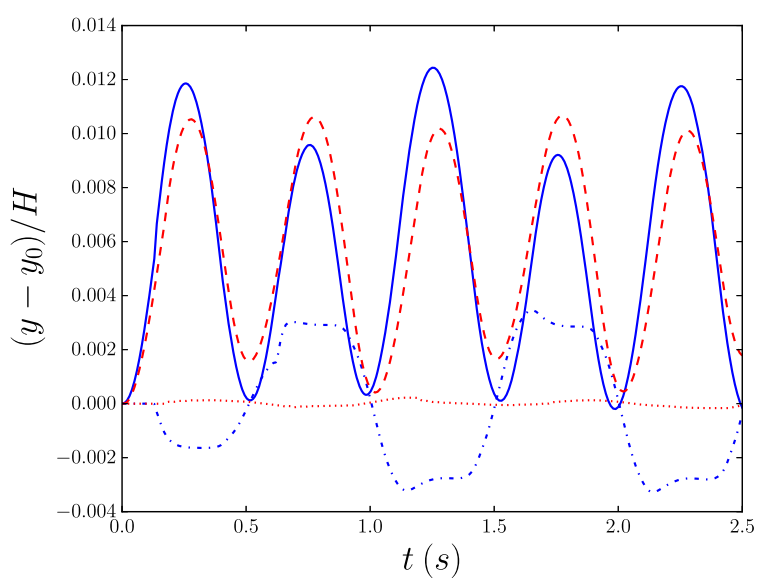

(b)

FIG. 6. Time history of displacements of $P_{1}$ and $P_{5}$ for the non-magnetic case with $\gamma_{0}=1.2 / \pi$ and $\omega=2 \pi \mathrm{rad} / \mathrm{s}$. 


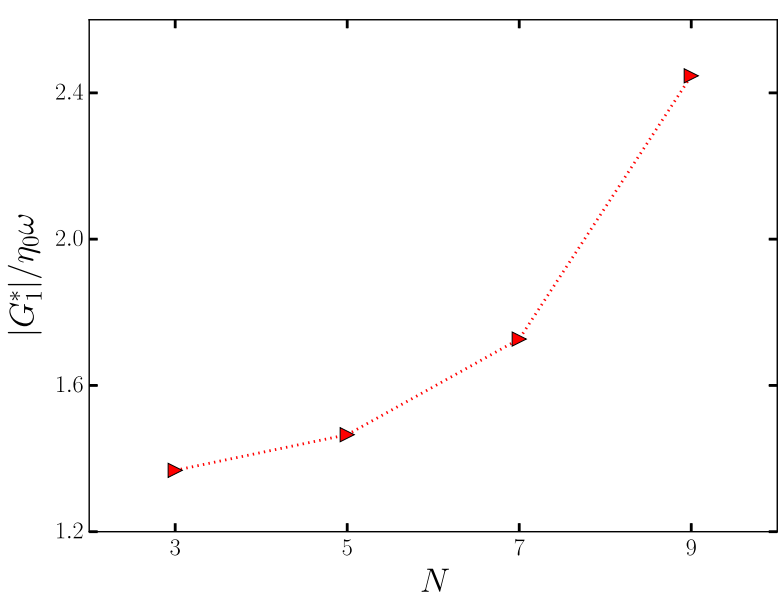

(a)

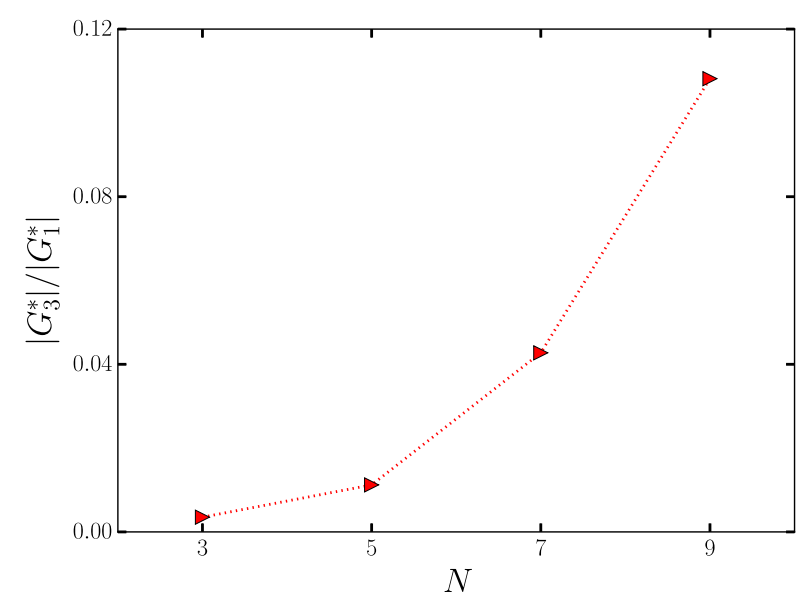

(b)

FIG. 7. (a) The non-dimensional strength of the first harmonic and (b) the intensity of the non-linearity in the stress response as functions of $N$. Results are obtained for $M n^{*}=0.419$ and $\omega=2 \pi \mathrm{rad} / \mathrm{s}$ with $\gamma_{0}=1.2 / \pi$.

is changed. In this way, the effects of the magnetic field on the rheological behaviour of the system can be thoroughly investigated. Frequency is $\omega=2 \pi \mathrm{rad} / \mathrm{s}$ and kept constant for all these cases. In Fig. 8, the normalized strength of the first harmonic and $\Psi_{1}$ are shown as functions of $\left(M n^{*}\right)^{-1}$ for three different strain amplitudes $\left(\gamma_{0}\right)$. As seen in this figure, the first harmonic of the stress response is only slightly affected by increasing the strain amplitude in the range of $\gamma_{0}=0.05 / \pi$ to $0.4 / \pi$, while a significant change is observed by increasing it to $\gamma_{0}=1.2 / \pi$. This is a sign of an almost linear response for $\gamma_{0}<0.4 / \pi$.

The trends of variation of $\left|G_{1}^{*}\right|$ and $\Psi_{1}$ with $\left(M n^{*}\right)^{-1}$ (which is proportional to $B_{0}^{2}$ ) are almost similar for both the linear and non-linear regimes as shown in Fig. 8. The phase angle decreases by increasing the strength of the external magnetic field, and for moderate to high magnetic field strengths, the complex modulus is an increasing function of $\left(M n^{*}\right)^{-1}$. However, the slope of variations is smaller for a larger strain amplitude. This can be explained as a result of the larger contribution of the hydrodynamic forces in the effective rheology of the system. It is worth noting that for $\left(M n^{*}\right)^{-1}<$ 2.385 , the complex modulus, $\left|G_{1}^{*}\right|$, increases by increasing $\gamma_{0}$, while for $\left(M n^{*}\right)^{-1}>2.385,\left|G_{1}^{*}\right|$ reduces. Also, the phase angle, $\Psi_{1}$, is raised by increasing the strain amplitude although more significantly at larger $\left(M n^{*}\right)^{-1}$.

The corresponding elastic modulus, $G^{\prime}$, and dynamic viscosity, $\eta^{\prime}$, are shown in Fig. 9 as functions of $\left(M n^{*}\right)^{-1}$. As previously discussed, ${ }^{36}$ both $G^{\prime}$ and $\eta^{\prime}$ are increasing functions of $\left(M n^{*}\right)^{-1}$ (or equivalently $B_{0}$ ); however, it is observed in Fig. 9 that the slope is reduced by increasing the strain amplitude. In addition, it is noticeable that $G^{\prime}$ decreases by increasing $\gamma_{0}$, while $\eta^{\prime}$ increases. One should remember that $G^{\prime}$ and $\eta^{\prime}$ correspond to the first harmonic in the stress response.

In Fig. 10, the ratio $\left|G_{3}^{*}\right| /\left|G_{1}^{*}\right|$ and the phase angle of the third harmonic in the stress response are shown as functions of $\left(M n^{*}\right)^{-1}$ for $\gamma_{0}=0.4 / \pi$ and $1.2 / \pi$. It must be mentioned that non-linearities are negligible for $\gamma_{0}=0.05 / \pi$.

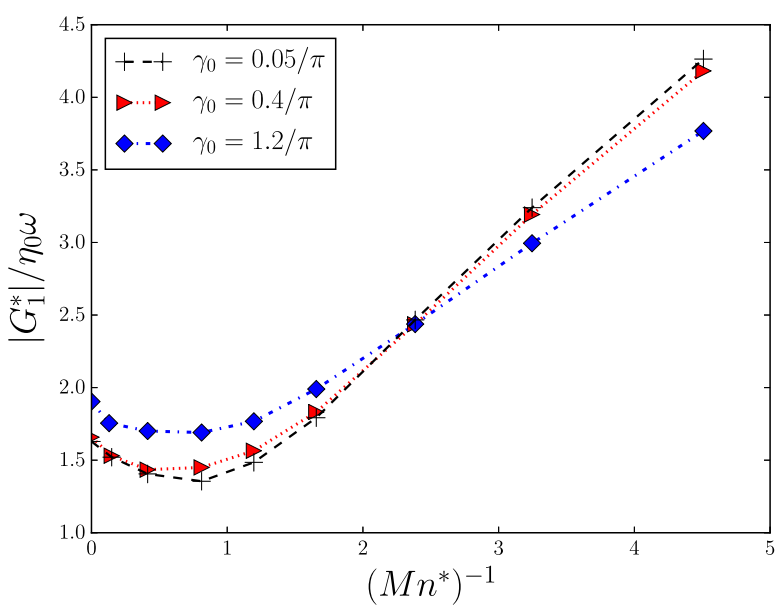

(a)

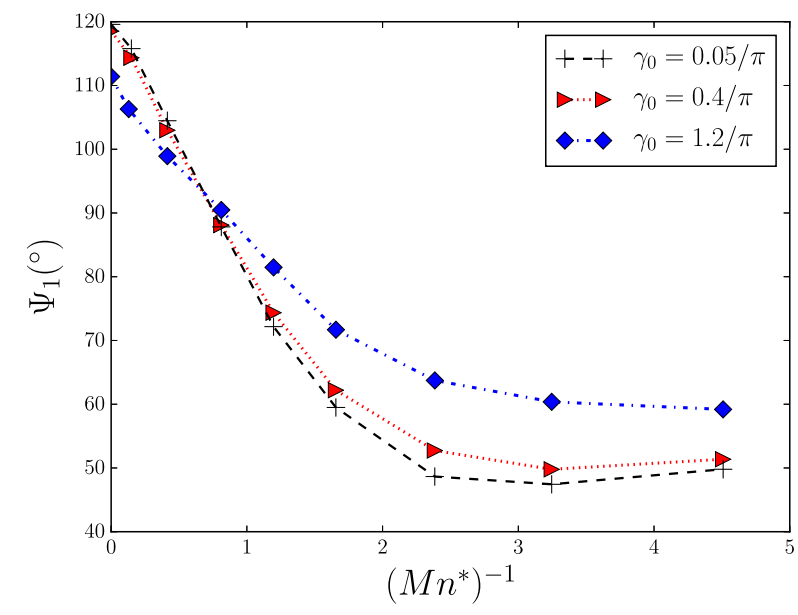

(b)

FIG. 8. (a) The normalized complex modulus, $\left|G_{1}^{*}\right| / \eta_{0} \omega$, and (b) the phase angle, $\Psi_{1}$, as functions of $\left(M n^{*}\right)^{-1}$ for $\omega=2 \pi \mathrm{rad} / \mathrm{s}$ and different strain amplitudes. 


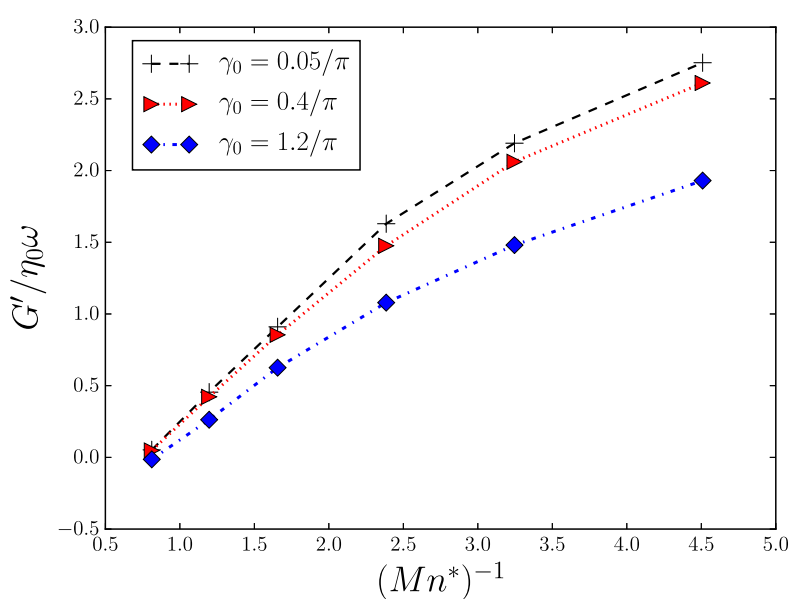

(a)

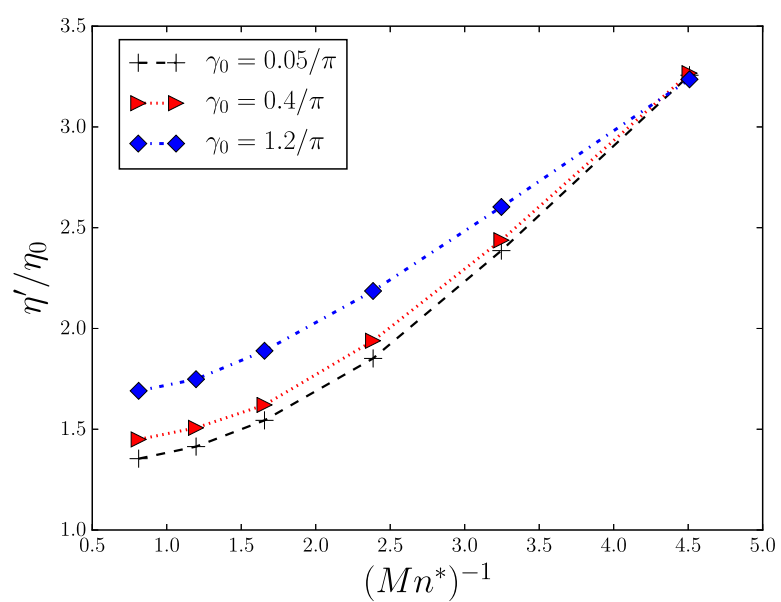

(b)

FIG. 9. (a) The elastic modulus and (b) dynamic viscosity as functions of $\left(M n^{*}\right)^{-1}$. Results are obtained for $\omega=2 \pi \mathrm{rad} / \mathrm{s}$ and different strain amplitudes.

For a smaller strain amplitude, $\gamma_{0}=0.4 / \pi$, by increasing $\left(M n^{*}\right)^{-1}$, first, the nonlinearity in the stress response becomes more significant, and then, a decreasing trend is observed for $\left(M n^{*}\right)^{-1}>0.812$. However, for $\gamma_{0}=1.2 / \pi$, the strength of the nonlinearity varies in a non-monotonic manner, i.e., there is a second local peak in the ratio $\left|G_{3}^{*}\right| /\left|G_{1}^{*}\right|$ occurring between $\left(M n^{*}\right)^{-1}=2.385$ and 3.246. Nevertheless, for both strain amplitudes, with $\left(M n^{*}\right)^{-1}>0.149$, the phase angle of the third harmonic is a monotonically increasing function of $\left(M n^{*}\right)^{-1}$.

The quality of the non-linear response of the system can be visualized by plotting variations of $\sigma^{\prime}$ and $\sigma^{\prime \prime}$ in a complete strain-cycle as shown in Fig. 11. As discussed in Ref. 36, it is expected that the slope of the curves presented in Figs. 11(a) and 11(b) shows an increasing trend with respect to the intensity of the external magnetic field. Although these results show an overall increase of the tangent moduli by increasing $\left(M n^{*}\right)^{-1}$, the intra-cycle behaviour of the system is extremely non-linear. The tangent elastic modulus presents a complex non-monotonic behaviour in a straincycle for a rather small magnetic flux density, while for a relatively strong magnetic field, $\left(M n^{*}\right)^{-1} \geq 3.246$ in this case, a strain-softening rheology (reduction of $\sigma^{\prime}$ by increasing $\gamma$ ) is observed [see Fig. 11(a)]. As seen in Fig. 11(b), for $\left(M n^{*}\right)^{-1}<3.246$, the current system behaves as a shear-thinning material. This behaviour changes by increasing $\left(M n^{*}\right)^{-1}$ and, ultimately, an intra-cycle shear-thickening behaviour (an increase in $\sigma^{\prime \prime}$ by increasing $\dot{\gamma}$ ) is observed for $\left(M n^{*}\right)^{-1}>3.246$.

The third coefficients of the Chebyshev representations of $\sigma^{\prime}$ and $\sigma^{\prime \prime}$ are shown in Fig. 12 as functions of $\left(M n^{*}\right)^{-1}$ for $\gamma_{0}=0.4 / \pi$ and $1.2 / \pi$. As seen in Fig. 12(a), the third elastic Chebyshev coefficient is a decreasing function of the magnetic flux density for $\left(M n^{*}\right)^{-1} \leq 3.246$. Here, a positive $e_{3}$ is obtained for $\left(M n^{*}\right)^{-1}<1.197$ that certifies a strain-stiffening (increasing slope $d \sigma^{\prime} / d \gamma$ by increasing $|\gamma|$ ) behaviour in a cycle. For $\left(M n^{*}\right)^{-1}>1.197, e_{3}$ becomes negative and gains a larger absolute value by further increasing $\left(M n^{*}\right)^{-1}$.

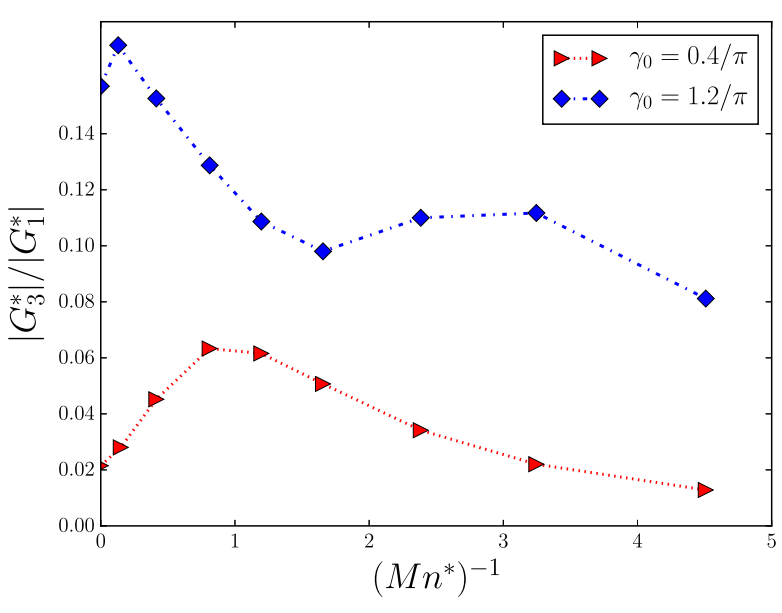

(a)

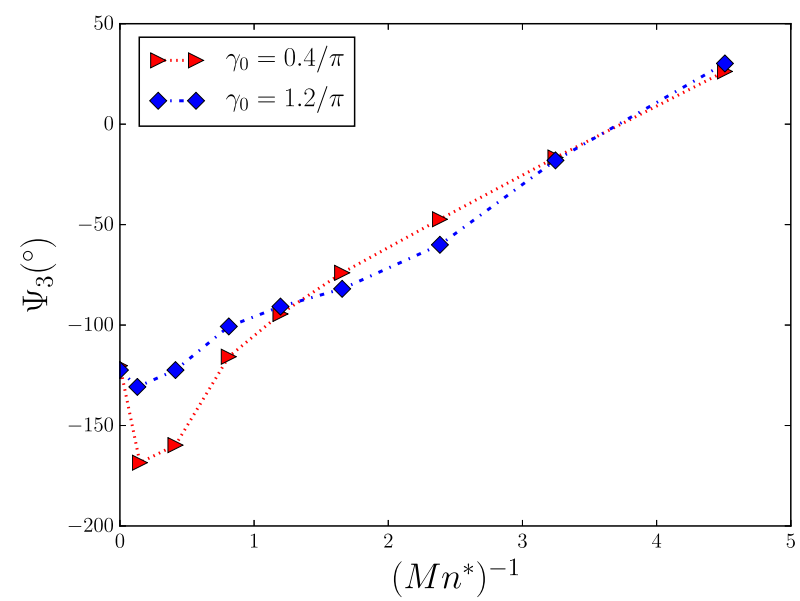

(b)

FIG. 10. (a) Normalized strength, $\left|G_{3}^{*}\right| /\left|G_{1}^{*}\right|$, and (b) the phase angle, $\Psi_{3}$, of the third harmonic in the stress response as functions of $\left(M n^{*}\right)^{-1}$ for $\omega=2 \pi$ and different strain amplitudes. 


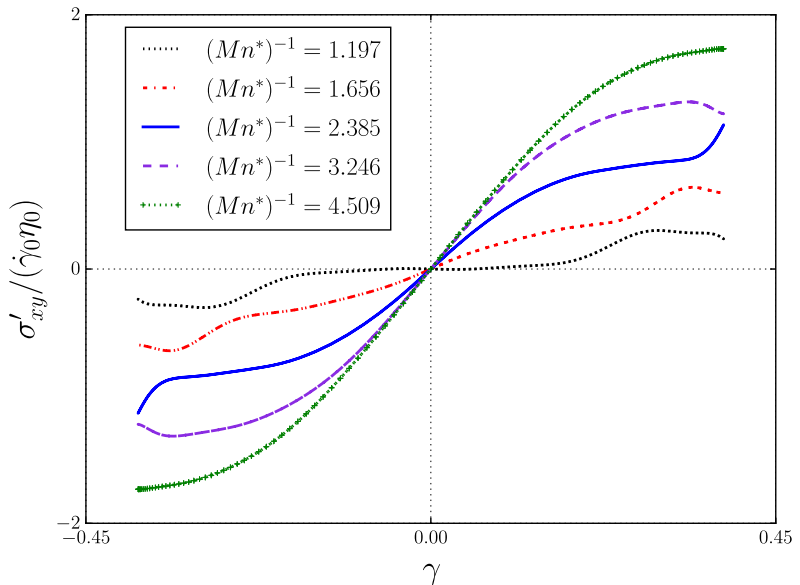

(a)

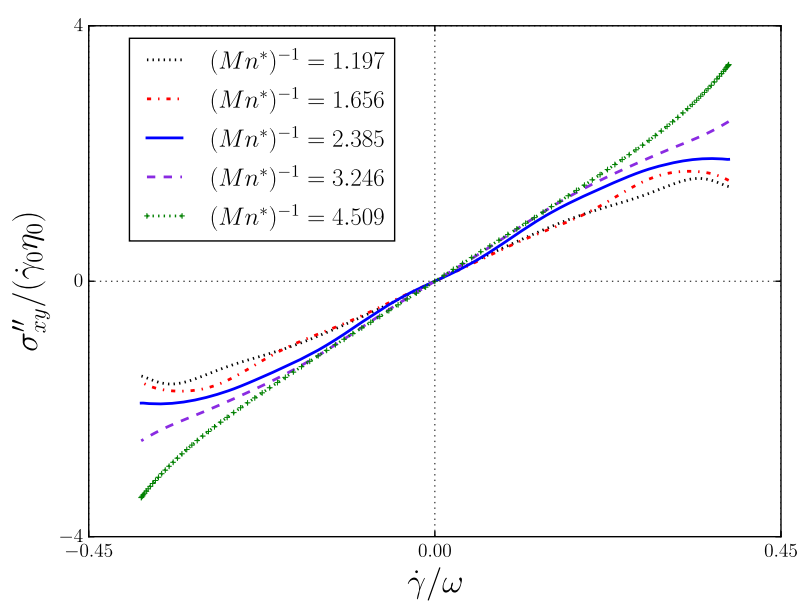

(b)

FIG. 11. The intra-cycle variation of (a) the elastic stress and (b) the viscous stress obtained for $\gamma_{0}=1.2 / \pi$ and different $\left(M n^{*}\right)^{-1}$, with $\omega=2 \pi \mathrm{rad} / \mathrm{s}$.

This leads to the strain-softening (decreasing slope $d \sigma^{\prime} / d \gamma$ by increasing $|\gamma|$ ) behaviour observed in Fig. 11(a). The increasing trend of the third elastic Chebyshev coefficient for $\left(M n^{*}\right)^{-1}>3.246$ can be partially due to a decrease in $\left|G_{3}^{*}\right|$ as seen in Fig. 10(a). With $\gamma_{0}=0.4 / \pi$ and $1.2 / \pi$, the third viscous Chebyshev coefficient shows an increasing trend for $\left(M n^{*}\right)^{-1} \geq 1.197$ and $\left(M n^{*}\right)^{-1} \geq 2.385$, respectively. The coefficient $v_{3}$ is negative for approximately $\left(M n^{*}\right)^{-1}<3.726$. This leads to an intra-cycle shear-thinning behaviour as seen in Fig. 11(b). By further increasing $\left(M n^{*}\right)^{-1}$, ultimately, $v_{3}$ reaches a positive value for $\left(M n^{*}\right)^{-1}>3.726$ and the system behaves as a shear-thickening material [see Fig. 11(b)].

Comparing the absolute value of the third Chebyshev coefficients shown in Fig. 12 with $G^{\prime}$ and $\eta^{\prime}$ presented in Fig. 9, it is clear that the viscoelastic rheology of the system, especially for $\gamma_{0}=1.2 / \pi$, can be studied only by incorporating both the first and third harmonics of the stress response. Generally, the tangent elastic modulus at zero strain and the dynamic viscosity at zero strain-rate are good measures of the overall rheology of a system tested under LAOS. ${ }^{41}$

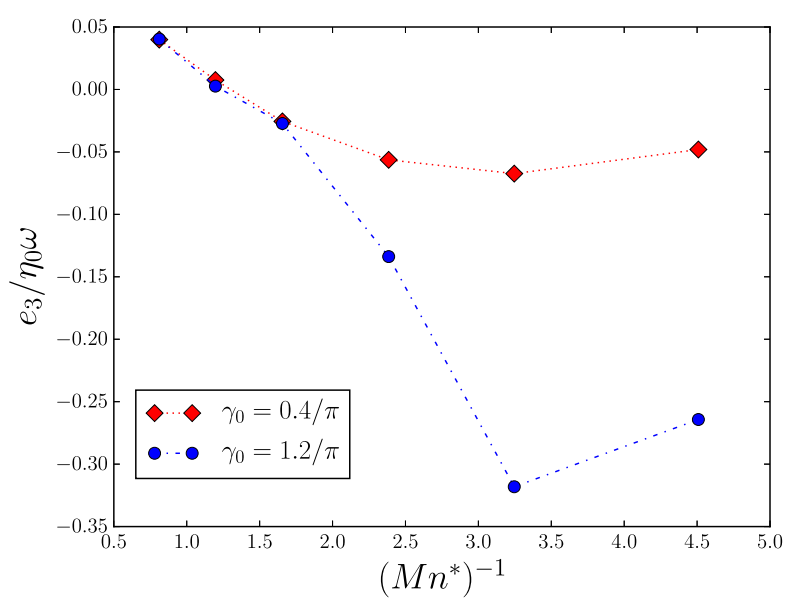

(a)
These parameters are approximated as $G_{M}^{\prime} \approx e_{1}-3 e_{3}$ and $\eta_{M}^{\prime} \approx v_{1}-3 v_{3}$ and shown in Fig. 13 as functions of $\left(M n^{*}\right)^{-1}$. As expected from previous discussions, ${ }^{36} G_{M}^{\prime}$ is a monotonically increasing function of $\left(M n^{*}\right)^{-1}$ (or equivalently $B_{0}$ ). However, the interesting point is that $G_{M}^{\prime}$ is almost independent of the strain amplitude. This behaviour is studied in more detail for the strain sweep tests. On the other hand, $\eta_{M}^{\prime}$ presents a non-monotonic variation for $\gamma_{0}=1.2 / \pi$, while it monotonically increases by increasing $\left(M n^{*}\right)^{-1}$ for $\gamma_{0}=0.4 / \pi$. Using Eqs. (15) and (16) and considering the absolute value of the parameters shown in Figs. 9(b) and 12(b), it can be inferred that $v_{3}$ plays an important role in determining the trend of variations of $\eta_{M}^{\prime}$.

\section{Micro-structure}

To learn more about the rheology of the present system, it is also worth investigating the micro-structure of the magnetic cluster under LAOS tests. In Fig. 14, the arrangement of the magnetic particles is shown at the moment when the magnetic chain reaches its largest tilting angle (or equivalently $P_{1}$

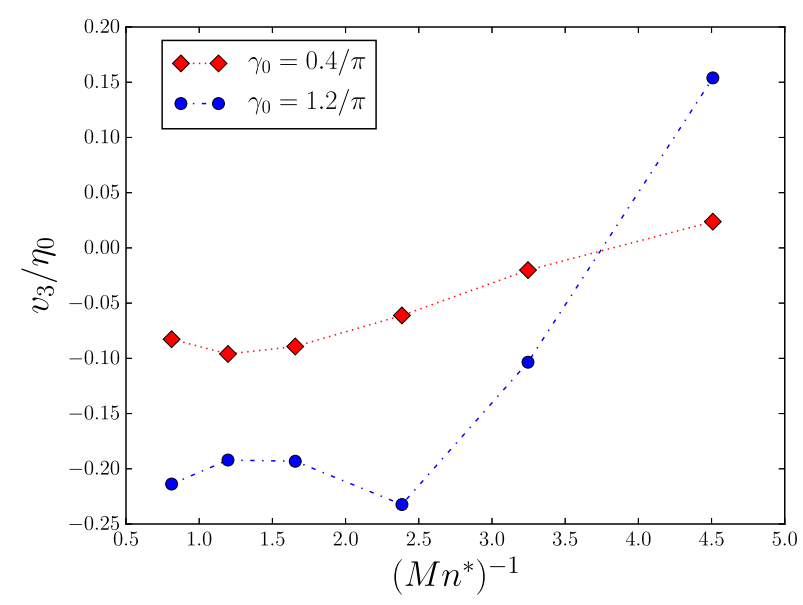

(b)

FIG. 12. Normalized third (a) elastic and (b) viscous Chebyshev coefficients as functions of $\left(M n^{*}\right)^{-1}$ obtained for $\omega=2 \pi$ rad/s and two different $\gamma_{0}$. 


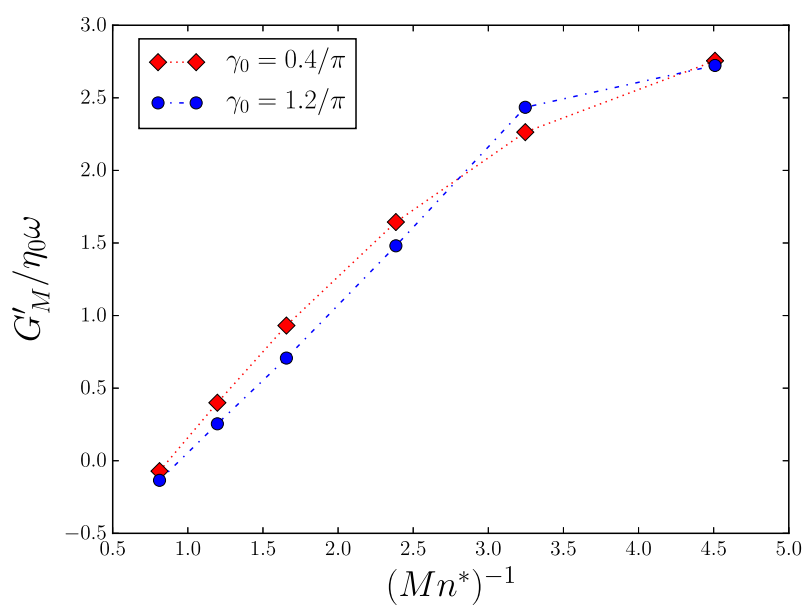

(a)

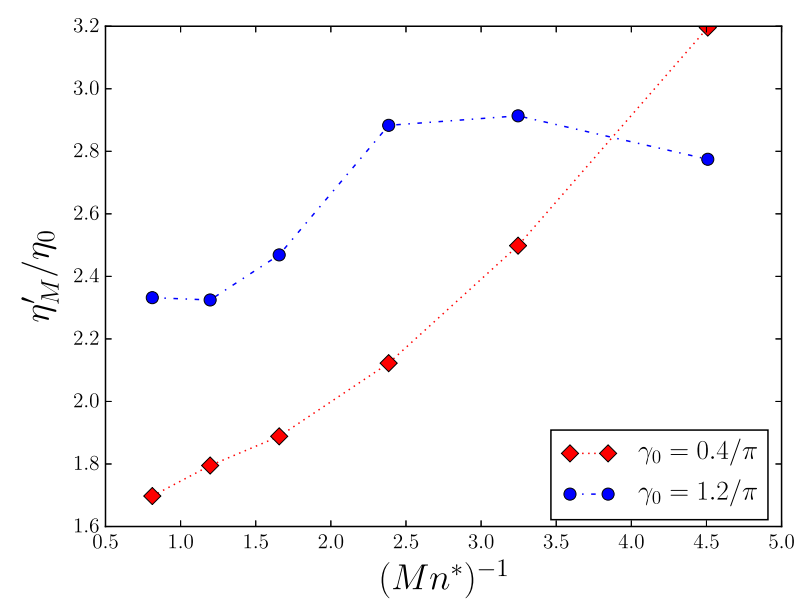

(b)

FIG. 13. Normalized (a) tangent elastic moduli at zero strain and (b) tangent dynamic viscosity at zero strain rate as functions of $\left(M n^{*}\right)^{-1}$ obtained for $\omega=2 \pi$ $\mathrm{rad} / \mathrm{s}$ and two different $\gamma_{0}$.

reaches its right most position). This occurs at different times for different $\left(M n^{*}\right)^{-1}$ with a constant frequency $(\omega=2 \pi \mathrm{rad} / \mathrm{s})$ and the same strain amplitude $\left(\gamma_{0}=1.2 / \pi\right)$. It is obvious that the stronger the external magnetic field [larger $\left(M n^{*}\right)^{-1}$ ], the stiffer the magnetic chain and the smaller the tilting angle. Therefore, by increasing $\left(M n^{*}\right)^{-1}$, the stress response is intensified as observed in Fig. 8(a). Moreover, both the elasticity and viscosity are expected to be larger for a stiffer magnetic chain which is in agreement with Fig. 9. Theoretical mod$\mathrm{els}^{31}$ that have been developed for a steady shear test also predict a similar behaviour, i.e., a reduction in the tilting angle as well as an increase in the shear stress by increasing the magnetic field intensity. However, a quantitative comparison with theory needs a model that is particularly developed for

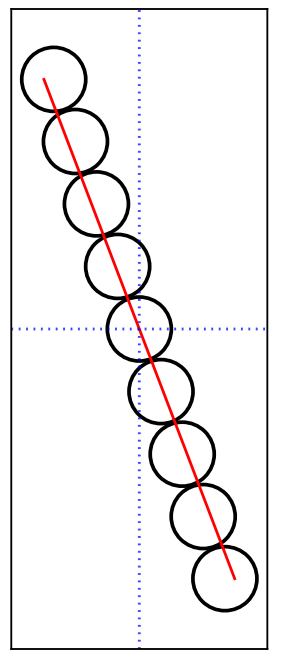

(a)

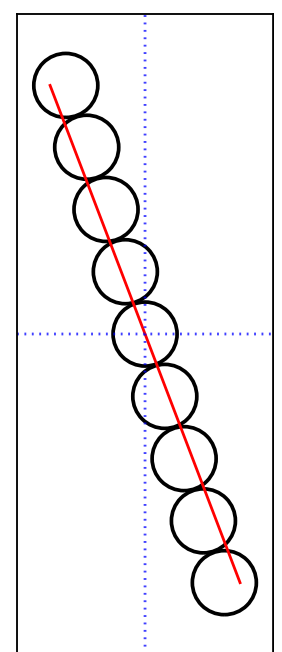

(b)

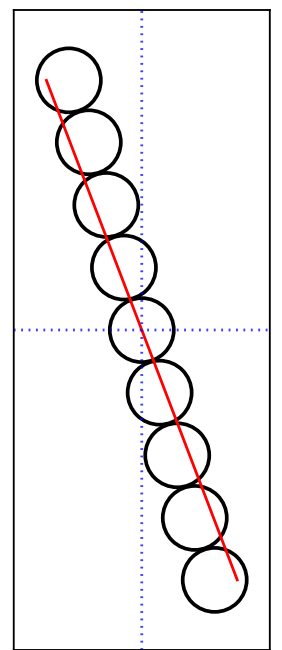

(c)
FIG. 14. The arrangement of solid particles once the magnetic chain reaches the largest tilting angle. The solid line shows the maximum displacement in the absence of solid particles in line with the input strain amplitude. Results are obtained for $\gamma_{0}=1.2 / \pi$ and $\omega=2 \pi \mathrm{rad} / \mathrm{s}$ and different $\left(M n^{*}\right)^{-1}$. (a) $\left(M n^{*}\right)^{-1}$ $=2.385, \omega t=1.40 \pi$. (b) $\left(M n^{*}\right)^{-1}=3.246, \omega t=1.34 \pi$. (c) $\left(M n^{*}\right)^{-1}=4.509$, $\omega t=1.30 \pi$. an oscillatory shear test and circular magnetic particles in two dimensions.

However, it is more suitable to quantify the arrangement of solid particles using the non-dimensional slope defined as

$$
\bar{s}_{x}=\frac{1}{\gamma_{0}} \frac{x-L / 2}{y-H / 2} .
$$

Figure 15 presents $\bar{s}_{x}$ for the four solid particles positioned at the bottom half of the magnetic cluster. In this figure, $\bar{y}=y / H$ and $P_{1}(\bar{y} \approx 0.1)$ has reached its maximum lateral position. As seen in this figure, generally, the slope decreases by increasing the amplitude of the input strain. However, by decreasing $\left(M n^{*}\right)^{-1}$, this variation also becomes smaller; beyond $\gamma_{0}=0.8 / \pi$, the variation in the slope is small for $\left(M n^{*}\right)^{-1}=$ 2.385, while for $\left(M n^{*}\right)^{-1}<2.385$, it is negligible compared to the results obtained for larger $\left(M n^{*}\right)^{-1}$.

In a similar way as the non-dimensional slope defined in Eq. (17), the velocity of the solid particles can also be quantified using the non-dimensional velocity defined as

$$
\bar{s}_{v}=\frac{1}{\dot{\gamma}_{0}} \frac{v_{x}}{y-H / 2} .
$$

Figure 16 demonstrates $\bar{s}_{v}$ for the four solid particles positioned at the bottom half of the magnetic chain once the input strain-rate reaches its peak $\left(\dot{\gamma}=\dot{\gamma}_{0}\right)$. As seen in Fig. 15 for $\bar{s}_{x}$, by increasing the amplitude of the input strain, $\bar{s}_{v}$ also varies. However, unlike $\bar{s}_{x}$, the trend in the variation of $\bar{s}_{v}$ is not similar for all $\left(M n^{*}\right)^{-1}$; for $\left(M n^{*}\right)^{-1} \leq 2.385$, the variation in the non-dimensional velocity of $P_{1}$ (positioned at $\bar{y} \approx 0.1)$ is negligible, while $\bar{s}_{v}$ increases for the upper solid particles which are nearer to the center of the chain. For $\left(M n^{*}\right)^{-1}=3.246$, the variation in $\bar{s}_{v}$ is small, while for the strongest magnetic field with $\left(M n^{*}\right)^{-1}=4.509, \bar{s}_{v}$ shows a decreasing trend for all solid particles. Such a change in the micro-structural behaviour of the system signifies that its nonlinear rheological behaviour would also become substantially different as the Mason number is changed. 


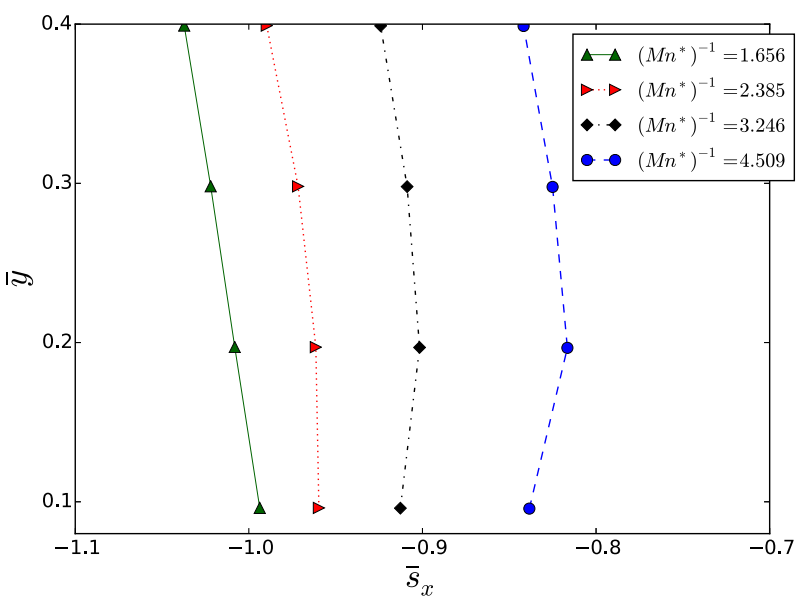

(a)

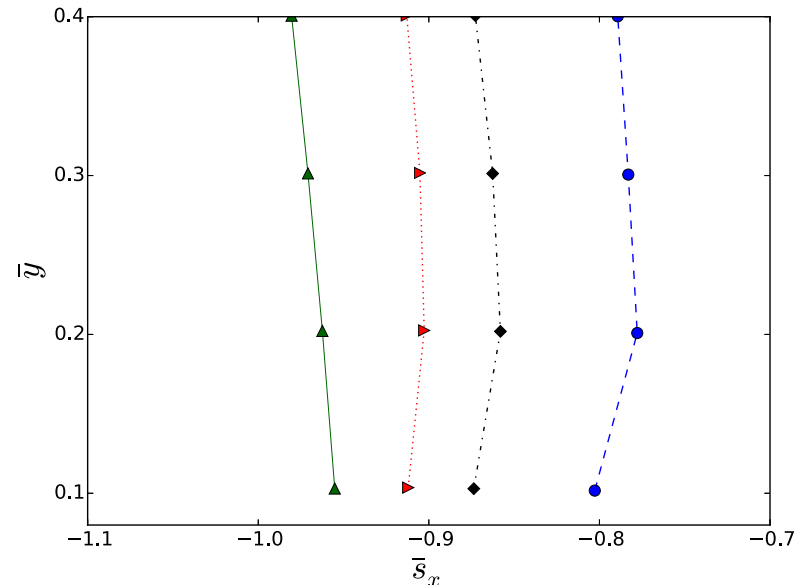

(b)

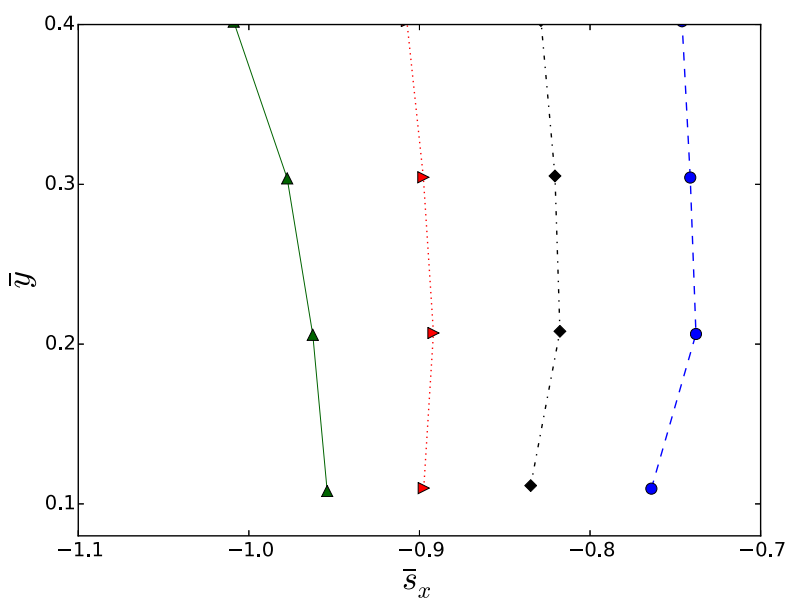

(c)

FIG. 15. Non-dimensional slope obtained for the four solid particles positioned at the bottom of the magnetic chain. Results are plotted at the moment that the magnetic chain reaches the largest tilting angle and are obtained for $\omega=2 \pi \mathrm{rad} / \mathrm{s}$ and different $\left(M n^{*}\right)^{-1}$. (a) $\gamma_{0}=0.4 / \pi$. (b) $\gamma_{0}=0.8 / \pi$. (c) $\gamma_{0}=1.2 / \pi$.

\section{Strain sweep tests}

In the literature, ${ }^{41,53}$ it is discussed that the intra-cycle non-linear behaviour of a system obtained for a single strain amplitude cannot be generalized to its overall non-linear rheology. Therefore, strain sweep tests shall be utilized to study the overall rheology of the system ${ }^{41}$ beyond its intra-cycle behaviour. In this section, two sets of tests are conducted; in the first set, frequency is kept constant while the strain amplitude is changed, and in the second set, the amplitude of the strain-rate is kept constant while the frequency varies to adjust $\gamma_{0}$. Figure 17 illustrates the normalized complex modulus and phase angle corresponding to the first harmonic in the stress response as functions of $\gamma_{0}$. In these cases, frequency is $\omega=2 \pi \mathrm{rad} / \mathrm{s}$ and $\gamma_{0}$ is proportional to the strain-rate amplitude.

For the non-magnetic case, $\left|G_{1}^{*}\right|$ exhibits an increasing trend with $\gamma_{0}$; however, this is reversed for a rather large magnetic flux density $\left(M n^{*} \leq 0.308\right.$ in the present case). For $M n^{*}=$ 0.419 , the variation of $\left|G_{1}^{*}\right|$ with $\gamma_{0}$ is almost negligible. Considering the first harmonic as the most influential part of the stress response, it can be concluded that for a rather large $B_{0}$ $\left(\right.$ small $\left.M n^{*}\right)$, the stress response becomes weaker by increasing $\gamma_{0}$. This is due to the fact that the more the magnetic cluster is tilted, the weaker the magnetic bonds become. As discussed in Ref. 36, for $B_{0}=0$, inertia leads to an obtuse phase angle. This phase angle reduces by increasing $\gamma_{0}$. This reduction is associated with a reduction in the loss of energy. For all magnetic cases considered in this section, $\Psi_{1}$ is an increasing function of $\gamma_{0}$, which certifies a reduction in the elastic portion of the stress response. In Fig. 18, $G^{\prime}$ and $\eta^{\prime}$ are shown as functions of $\gamma_{0}$.

The elastic modulus is clearly a decreasing function of $\gamma_{0}$ while dynamic viscosity increases only slightly in the strain sweep test. However, the smaller the magnetic field intensity (larger $M n^{*}$ ), the larger is the variation of $\eta^{\prime}$ with $\gamma_{0}$. Nevertheless, $G^{\prime}$ and $\eta^{\prime}$ only represent the first harmonic in the stress response and the overall rheology of the system can be deduced by investigating the higher harmonics. The parameters which correspond to the third harmonic, $\left|G_{3}^{*}\right| /\left|G_{1}^{*}\right|$ and $\Psi_{3}$, are shown in Fig. 19 as functions of $\gamma_{0}$. It must be noted that higher harmonics are relatively insignificant in the overall stress response. As shown in Fig. 19(a), the non-linearity of the stress response generally becomes more significant by increasing the strain amplitude. Nevertheless, with a moderately small magnetic flux density, $M n^{*}=0.419$ 


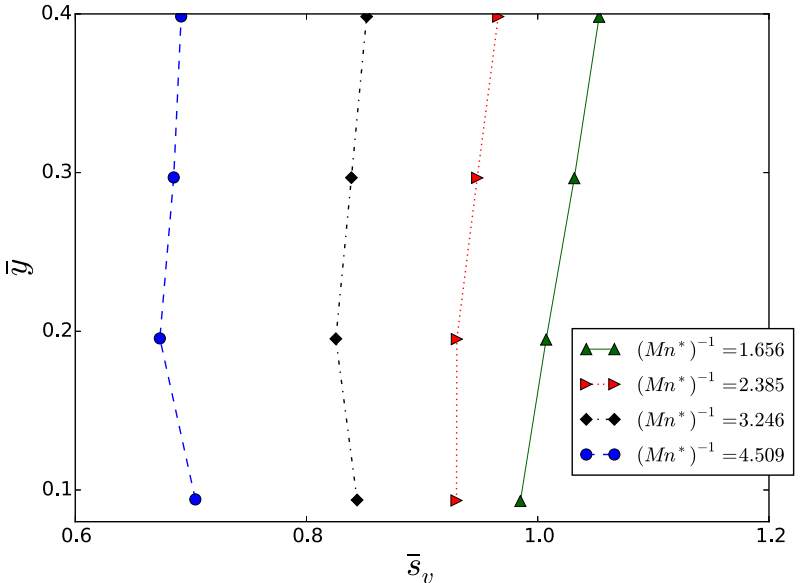

(a)

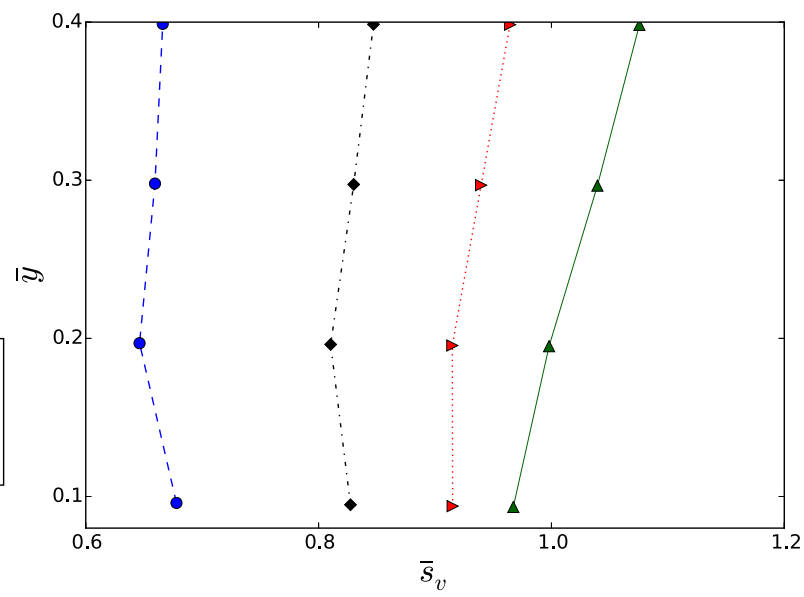

(b)

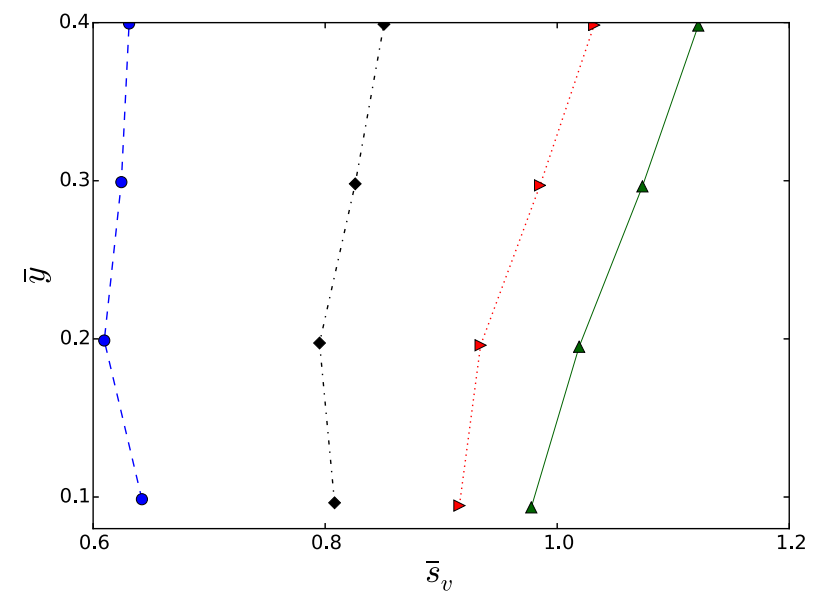

(c)

FIG. 16. Non-dimensional velocity obtained for the four solid particles positioned at the bottom of the magnetic chain. Results are obtained at $\omega t=2 \pi$ for $\omega=2 \pi \mathrm{rad} / \mathrm{s}$ and different $\left(M n^{*}\right)^{-1}$. (a) $\gamma_{0}=0.4 / \pi$. (b) $\gamma_{0}=0.8 / \pi$. (c) $\gamma_{0}=1.2 / \pi$.

in this case, the slope of the variation of $\left|G_{3}^{*}\right| /\left|G_{1}^{*}\right|$ with $\gamma_{0}$ is the largest for $\gamma_{0} \leq 0.25$, while beyond this range, i.e., $\gamma_{0}>0.25$, a remarkable decrease in the slope occurs.
For $\gamma_{0} \geq 0.1$, the phase angle of the third harmonic is almost independent of $\gamma_{0}$. For smaller strain amplitudes, the stress response is almost linear, and therefore, the

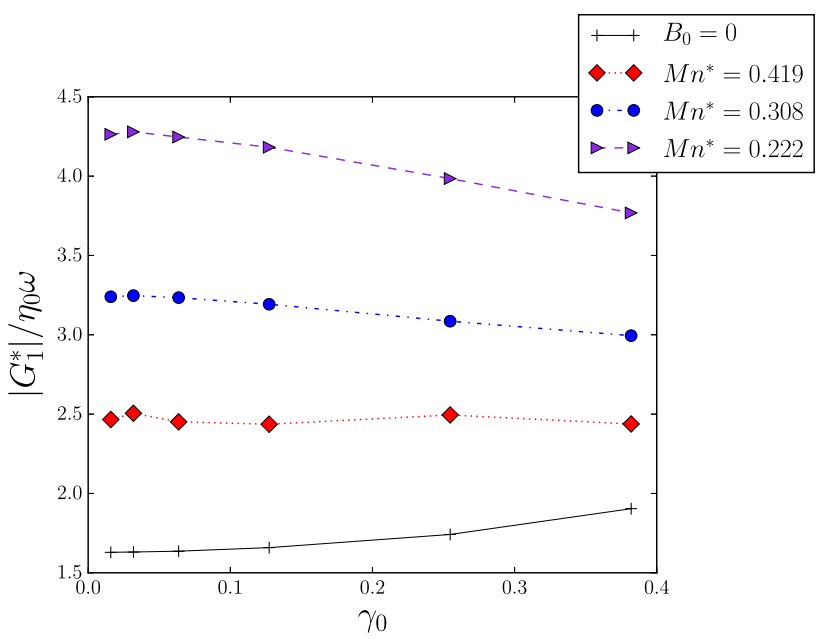

(a)

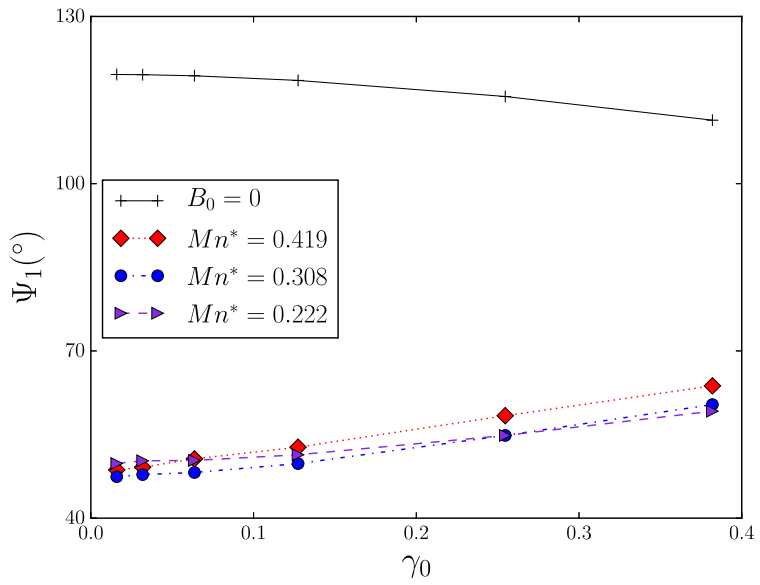

(b)

FIG. 17. (a) Normalized $\left|G_{1}^{*}\right|$ and (b) $\Psi_{1}$ as functions of $\gamma_{0}$. Results are obtained for $\omega_{0}=2 \pi \mathrm{rad} / \mathrm{s}$ and different $M n^{*}$. 


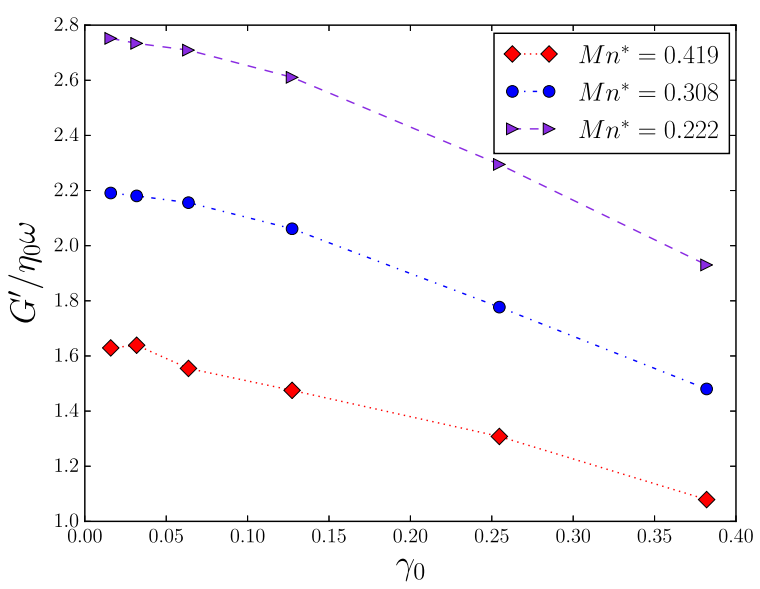

(a)

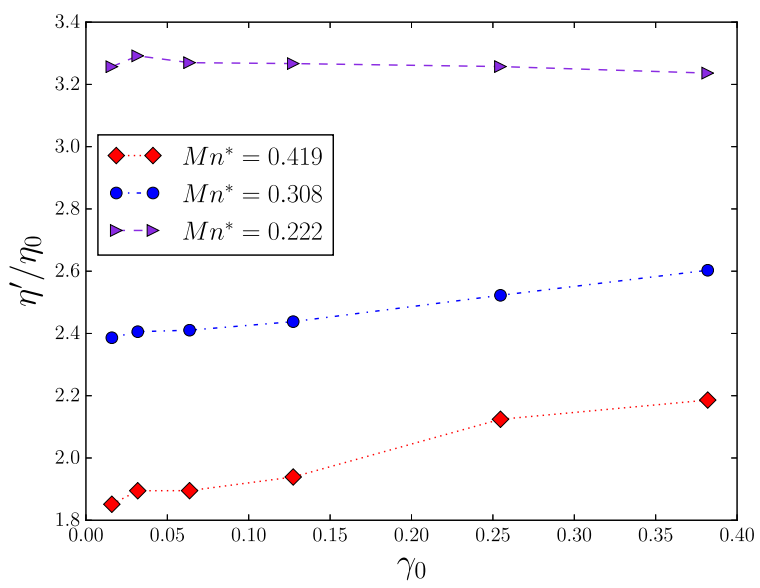

(b)

FIG. 18. (a) The elastic modulus and (b) dynamic viscosity as functions of $\gamma_{0}$. Results are obtained for $\omega_{0}=2 \pi \mathrm{rad} / \mathrm{s}$ and different $M n^{*}$.

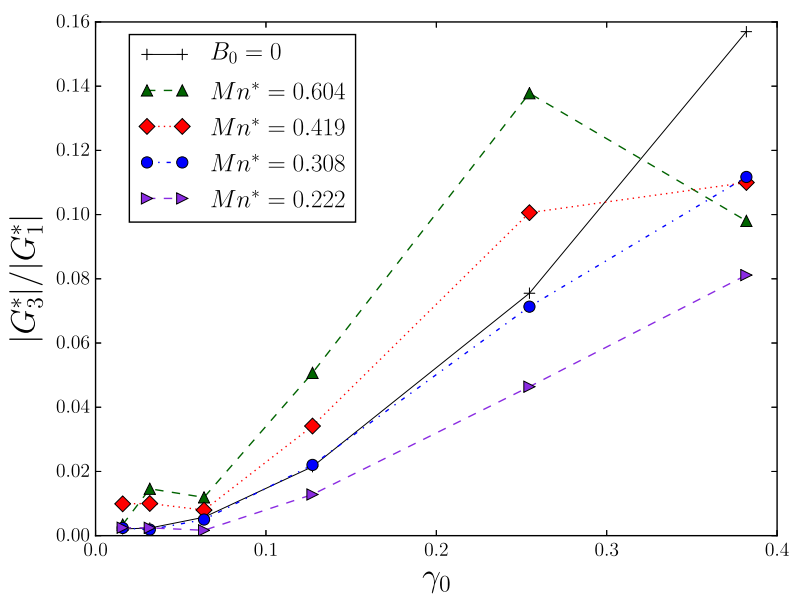

(a)

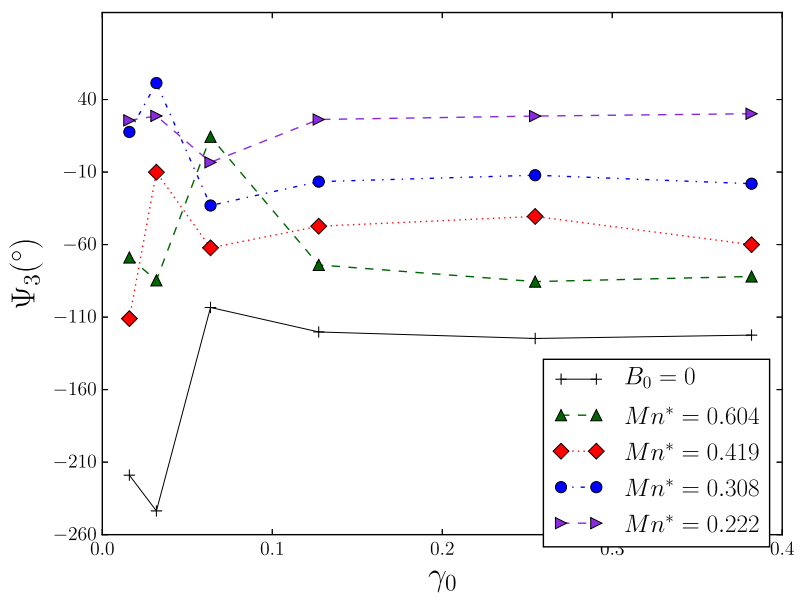

(b)

FIG. 19. (a) The intensity of the third harmonic and (b) $\Psi_{3}$ as functions of $\gamma_{0}$. Results are obtained for $\omega_{0}=2 \pi \mathrm{rad} / \mathrm{s}$ and different $M n^{*}$.

variation of $\Psi_{3}$ has no significant effect on the rheology of the system.

In order to further investigate the non-linear intra-cycle behaviour of the system, the third Chebyshev coefficients of the elastic and viscous stress are shown in Fig. 20 as functions of the strain amplitude. For all cases presented in Fig. 20(a), the third elastic Chebyshev coefficient is negative and the system shows a strain-softening behaviour in a

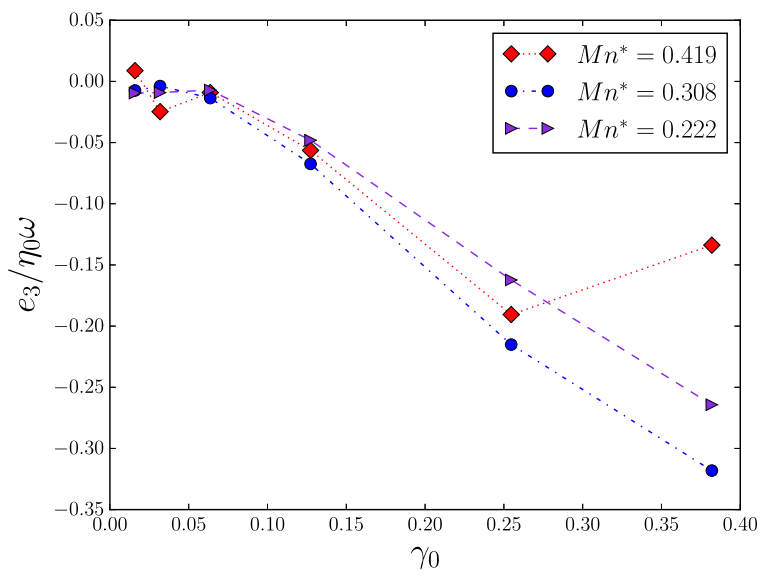

(a)

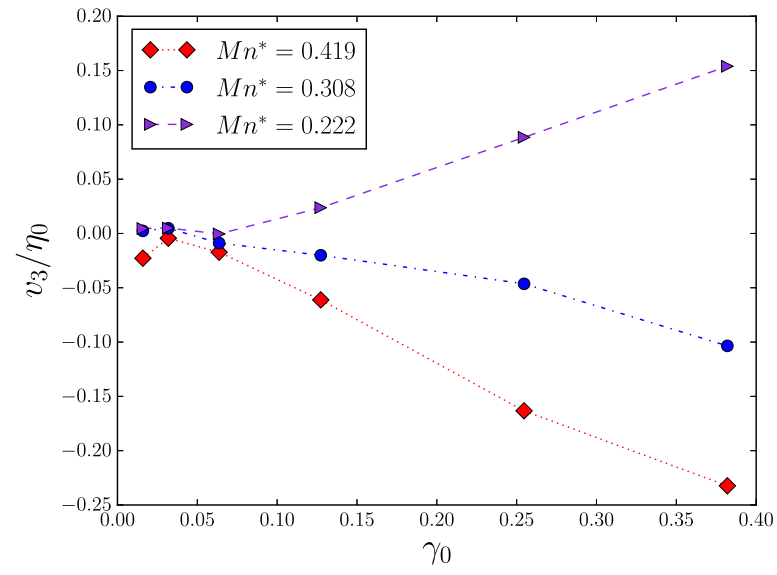

(b)

FIG. 20. Normalized third (a) elastic and (b) viscous Chebyshev coefficients as functions of $\gamma_{0}$. Results are obtained for $\omega_{0}=2 \pi \mathrm{rad} / \mathrm{s}$ and different $M n^{*}$. 


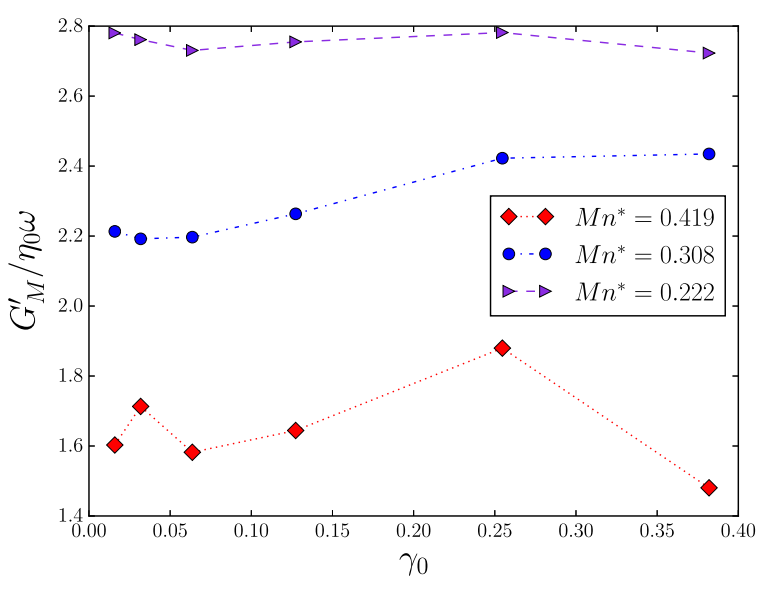

(a)

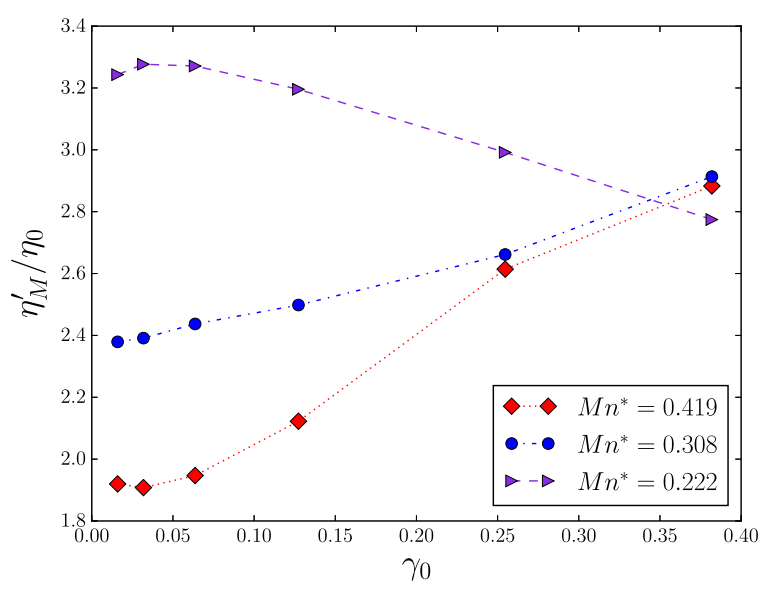

(b)

FIG. 21. Normalized (a) tangent elastic moduli at zero strain and (b) tangent dynamic viscosity at zero strain rate as functions of $\gamma_{0}$. Results are obtained for $\omega_{0}=2 \pi \mathrm{rad} / \mathrm{s}$ and different $M n^{*}$.

large-amplitude strain-cycle. Unless for $\gamma_{0}>0.25$ with $M n^{*}=0.419$, the absolute value of $e_{3}$ increases by increasing $\gamma_{0}$. This change in the trend of $e_{3}$ is associated with the sudden reduction in the slope of $\left|G_{3}^{*}\right| /\left|G_{1}^{*}\right|$ observed in Fig. 19(a). It is interesting that unlike $e_{3}$, the third viscous Chebyshev coefficient reflects different trends for different magnetic flux densities. With a moderately low magnetic field strength, $M n^{*}=0.419, v_{3}$ is negative and a decreasing function of $\gamma_{0}$, while for $M n^{*}=0.222, v_{3}$ increases by increasing the strain amplitude. This means that based on the strength of the external magnetic field, the system may exhibit either a shearthinning (for a moderately small $B_{0}$ ) or a shear-thickening behaviour (for a rather large $B_{0}$ ) in a large-amplitude strain-cycle.

Tangent elastic modulus at zero-strain and tangent dynamic viscosity at zero strain-rate are shown in Fig. 21 as functions of the strain amplitude. It was discussed above that for the present test cases, $G^{\prime}$ is a decreasing function of $\gamma_{0}$, while $\eta^{\prime}$ is almost independent of the strainamplitude. However, especially for $\gamma_{0}>0.1, e_{3}$ and $v_{3}$ play a

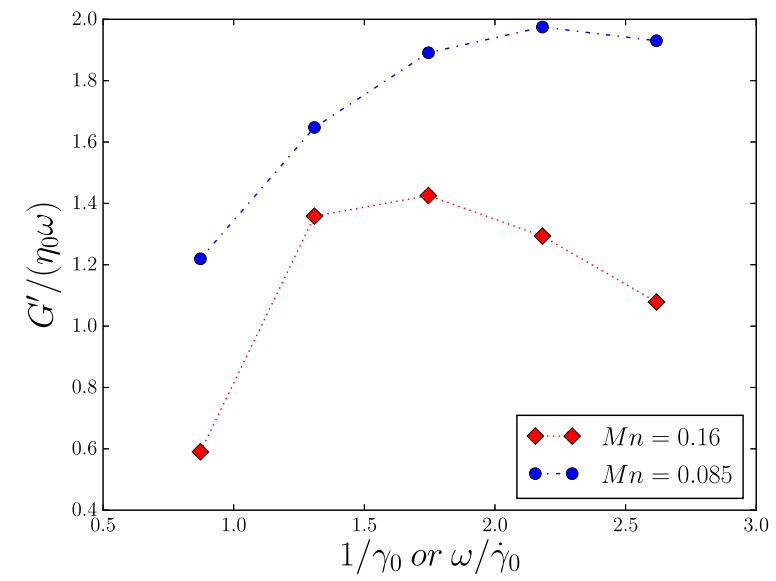

(a) remarkable role in the calculation of the tangent moduli. As seen in Fig. 21(a), despite the intra-cycle strain-softening behaviour of the system, $G_{M}^{\prime}$ is almost independent of $\gamma_{0}$ for a rather strong magnetic field. For $M n^{*}=0.419, G_{M}^{\prime}$ shows a non-monotonic trend with the strain amplitude; however, its variation is small. On the other hand, since $\eta^{\prime}$ is almost independent of $\gamma_{0}$ in these strain sweep tests, similar to $v_{3}$, the trend of $\eta_{M}^{\prime}$ depends on the magnetic field intensity. For a rather strong magnetic field, $\eta_{M}^{\prime}$ is a decreasing function of $\gamma_{0}$, while for $M n^{*}=0.419, \eta_{M}^{\prime}$ increases by increasing the strain amplitude.

It is discussed in the literature ${ }^{57}$ that in a LAOS test, the strain-rate amplitude as well as frequency are the time scales associated with the micro-structural deformation of the system. In the above test cases, $\dot{\gamma}_{0}$ varied with the strain amplitude, while frequency was constant. In the following, the strainrate amplitude is kept constant and the strain sweep test is conducted by adjusting the frequency. In this way, the frequency dependence of the non-linear rheology of the system can be understood. ${ }^{41,57}$ It must be noted that since in each case

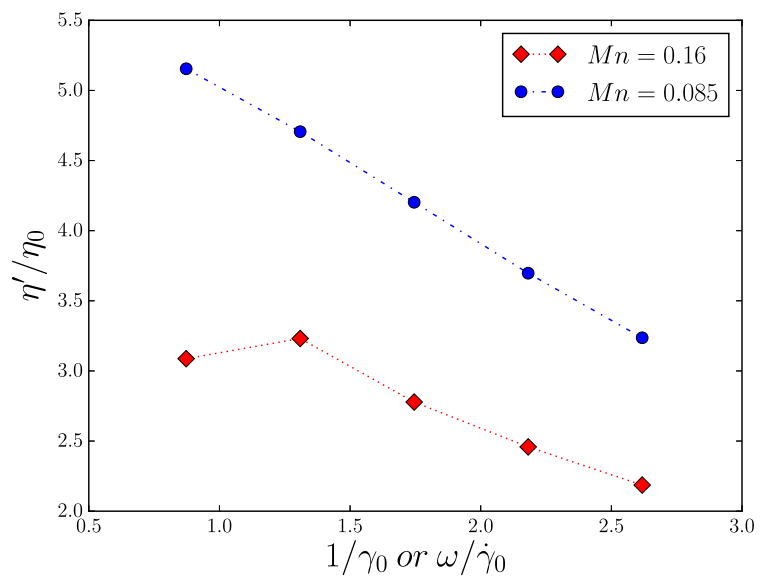

(b)

FIG. 22. (a) The elastic modulus and (b) dynamic viscosity as functions of $1 / \gamma_{0}$. Results are obtained for $\dot{\gamma}_{0}=2.4(1 / s)$ and two different Mason numbers. 
the amplitude of the strain-rate is constant and frequency is variable, the conventional definition of the Mason number (8) is used in order to non-dimensionalize the strength of the external magnetic field.

In Fig. 22, $G^{\prime}$ and $\eta^{\prime}$ are shown as functions of $\omega / \dot{\gamma}_{0}$ (or equivalently $1 / \gamma_{0}$ ) for a rather strong magnetic field, $M n=0.085$, and for a moderately small magnetic flux density, $M n=0.16$. For $M n=0.085$ within the range of $\gamma_{0}$ considered in this work, $G^{\prime}$ increases with $\omega$ and $\eta^{\prime}$ is a linearly decreasing function of frequency. However, for a rather large frequency, a decreasing trend is observed in Fig. 22(a) for $G^{\prime}$, which may be due to the intensified inertial effect. ${ }^{36}$ An almost similar trend is also observed for $M n=0.16$; however, the reduction in the elastic modulus is more severe. Another point to mention is the different trend of $\eta^{\prime}$ observed for a rather small frequency, $\omega=2 \pi / 3$ in the present case with $M n=0.16$. The unpredicted reduction in the dynamic viscosity is associated with the separation of the tip solid particles from the whole magnetic chain. This issue is shown in Fig. 23 which illustrates the arrangement of solid particles in the test domain at the moment that the magnetic chain has reached its maximum tilting angle. As expected, the smaller the frequency, the larger the chain deflection is. Also, it is known that magnetic bonds become weaker by increasing the angle formed between the magnetic chain and the direction of the external magnetic field. Therefore, there is a higher chance for particle separation at a rather small frequency as seen in Fig. 23(a) for $\omega=2 \pi / 3$. For $M n=0.085$, the magnetic field is strong enough to keep all particles in a single cluster even with $\omega=2 \pi / 3$. Nonetheless, the interesting point is that for the present test case, the separation occurs for the tip particles.

Figure 24 presents the ratio $\left|G_{3}^{*}\right| /\left|G_{1}^{*}\right|$ and $\Psi_{3}$ as functions of $\omega / \dot{\gamma}_{0}$ for the present test case. In Fig. 24(a), for $M n=0.085$, it is observed that the intensity of the third harmonic, as a measure of the non-linearity of the stress response, slightly decreases by increasing frequency (or equivalently decreasing $\gamma_{0}$ ). On the other hand for $M n=0.16$, the trend is non-monotonic. However, for both the Mason numbers, although the strain amplitude is changed in a rather wide range, $1.2 / \pi \leq \gamma_{0} \leq 3.6 / \pi,\left|G_{3}^{*}\right| /\left|G_{1}^{*}\right|$ varies only slightly

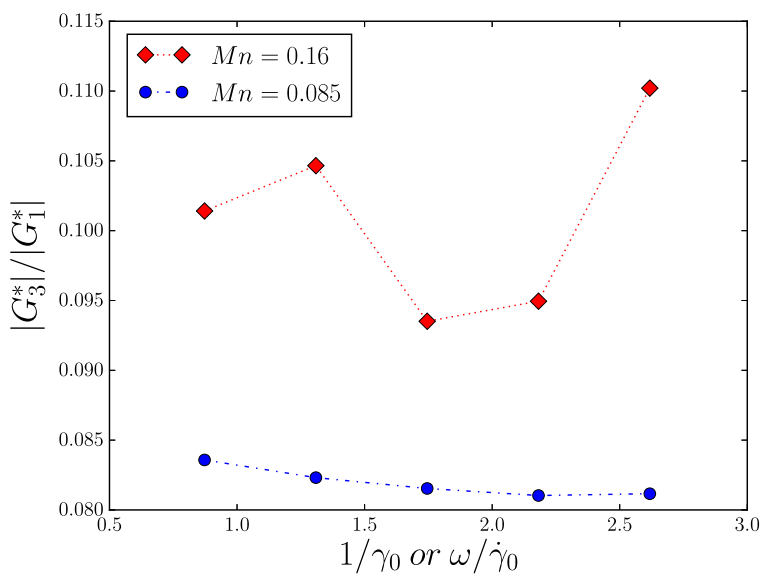

(a)

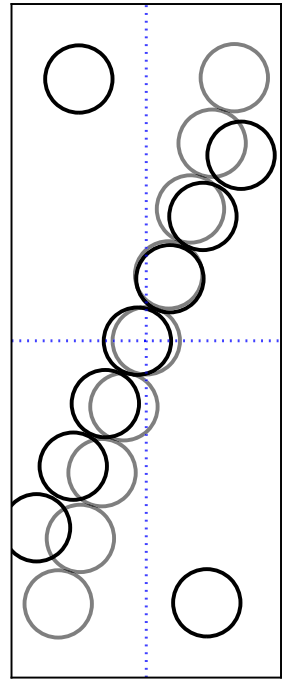

(a)

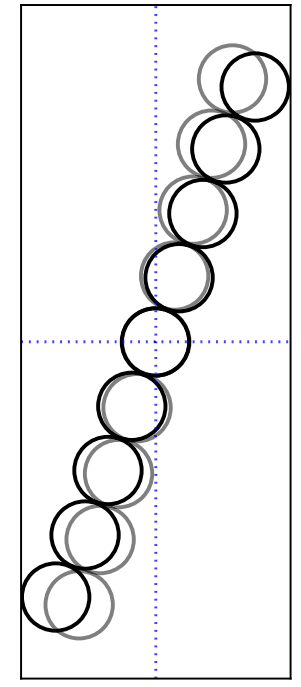

(b)
FIG. 23. Position of solid particles obtained for $\dot{\gamma}_{0}=2.4(1 / \mathrm{s})$ with (a) $M n=0.16$ and (b) $M n=0.085$. Results obtained for $\omega=2 \pi \mathrm{rad} / \mathrm{s}$ are shown with light color, while dark circles depict the cases with $\omega=2 \pi / 3 \mathrm{rad} / \mathrm{s}$.

by increasing $\omega$ as long as $\dot{\gamma}_{0}$ is constant. This underlines the fact that nonlinearities are more sensitive to the strain-rate amplitude than frequency. For both $M n$, as seen in Fig. 24(b), the phase angle of the third harmonic is a decreasing function of $\omega$.

Tangent elastic modulus at zero strain and tangent dynamic viscosity at zero shear-rate are shown in Fig. 25 as functions of $\omega / \dot{\gamma}_{0}$. Compared to the elastic modulus shown in Fig. 22(a), $G_{M}^{\prime}$ presents a similar trend but varies in a wider range by increasing $\omega$ while $\dot{\gamma}_{0}$ is constant. Here, $G_{M}^{\prime}$ is an increasing function of $\omega$; however, for $M n=0.16$, the slope gradually decreases and ultimately a decreasing function is observed in Fig. 25(a). This may be partially due to the inertia which becomes more effective at a rather large frequency and tend to decrease the effective elasticity of the system. ${ }^{36}$ On the other hand, compared to the dynamic viscosity shown in Fig. 22(b), $\eta_{M}^{\prime}$ presents a completely different trend; for

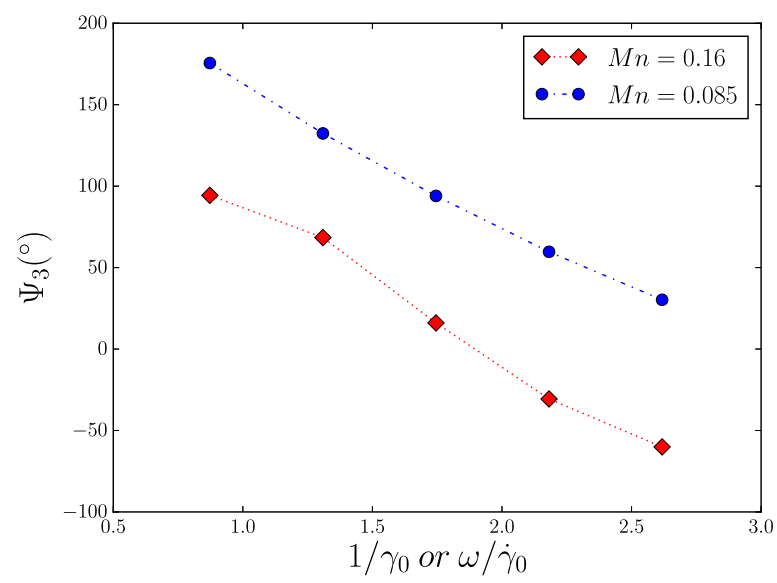

(b)

FIG. 24. (a) The intensity of the third harmonic and (b) $\Psi_{3}$ as functions of $1 / \gamma_{0}$. Results are obtained for $\dot{\gamma}_{0}=2.4(1 / s)$ and two different Mason numbers. 


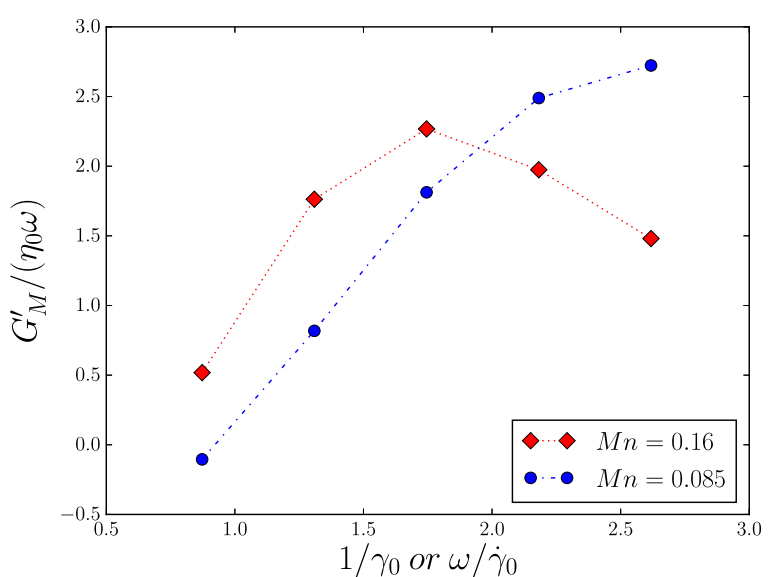

(a)

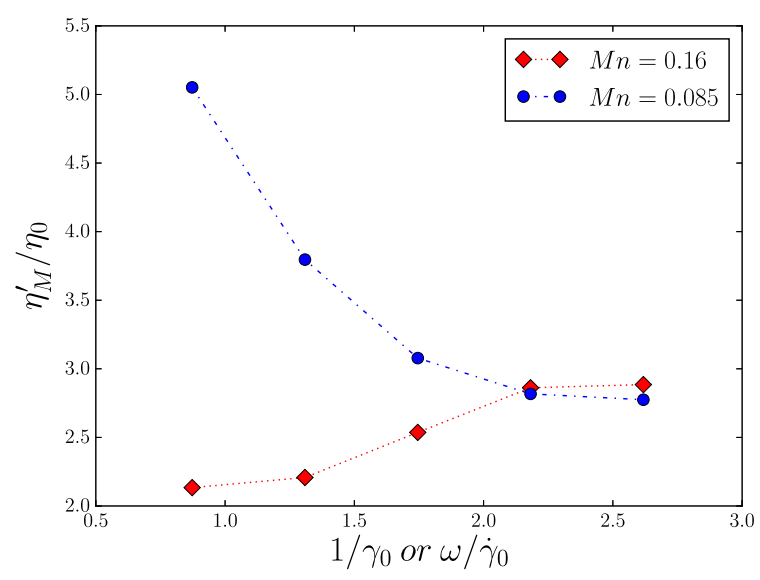

(b)

FIG. 25. Normalized (a) tangent elastic moduli at zero strain and (b) tangent dynamic viscosity at zero strain rate as functions of $1 / \gamma_{0}$. Results are obtained for $\dot{\gamma}_{0}=2.4(1 / s)$ and two different Mason numbers.

$M n=0.085$, it is a decreasing function of frequency, while for $M n=0.16, \eta_{M}^{\prime}$ slightly increases by increasing $\omega$.

\section{CONCLUSION}

In this paper, the nonlinear response of a periodic suspension of non-gap-spanning magnetic clusters was investigated using the LAOS tests. Results of the present work offer a new point of view in exploring the rheology of the magnetorheological fluids in the post-yield state by explaining the contribution of the non-gap-spanning clusters in the nonlinear viscoelastic behaviour of the system. Considering a symmetric configuration, disordered rearrangement of the magnetic clusters was avoided, and therefore, systematic analysis of the rheological response of this special microstructure was possible. The main concluding points are as follows:

- At rather small Mason numbers, the stronger the magnetic field, the smaller is the tilting angle of the cluster and the lower is the intensity of non-linearity.

- Both $G^{\prime}$ and $G_{M}^{\prime}$ were increasing functions of the intensity of the external magnetic field.

- While $\eta^{\prime}$ increased by increasing the intensity of the external magnetic field, $\eta_{M}^{\prime}$ showed a non-monotonic trend.

- The intra-cycle non-linear behaviour of the system was generally different from the non-linear rheology inferred from strain-sweep tests; for a rather weak magnetic field, a shear-thinning behaviour was observed during a strain cycle, while a shear-thickening behaviour was seen in the strain sweep test. On the other hand, for a rather strong magnetic field, the intra-cycle behaviour was shear-thickening, while a shear-thinning behaviour was observed in the strain sweep test.

- As long as no particle was detached from clusters, the intensity of the non-linear response was a function of the amplitude of the strain-rate (not the strain amplitude).
- Strain amplitude was responsible for particle separation which resulted in a significant reduction in the measured dynamic viscosity of the system.

- The system effectively exhibited a strain-softening behaviour, while the trend of dynamic viscosity strongly depended on the strength of the external magnetic field. The behaviour of the system changed from shear-thinning to shear-thickening by increasing the intensity of the magnetic field.

In this work, it was observed that under LAOS, particle separation occurs at the tips of a magnetic cluster. However, from a theoretical point of view, in a steady shear test with a uniform shear rate, chains are more prone to break at the center. ${ }^{31}$ In order to investigate this issue, theoretical models, e.g., see Ref. 31, are needed to be further developed to account for an oscillatory shear flow regime where the magnetic clusters are subject to non-affine ${ }^{34}$ time-dependent deformations with a phase different from the input strain. Such a model also facilitates the establishment of correlations between the microstructural parameters ( $\bar{s}_{x}$ and $\bar{s}_{v}$ as proposed in this work) and the physical properties of the system and may help explain the rheological behaviours observed in the present work. In this regard, beyond the hydrodynamic and magnetic forces, inertia may also have a substantial role.

\section{ACKNOWLEDGMENTS}

The corresponding author wishes to express his sincerest thanks to the Iran National Science Foundation (INSF) for supporting this work under Contract Number 92021291.

${ }^{1}$ D. J. Klingenberg and C. F. Zukoski IV, "Studies on the steady-shear behavior of electrorheological suspensions," Langmuir 6, 15-24 (1990).

${ }^{2}$ M. R. Jolly, J. D. Carlson, and B. C. Munoz, "A model of the behaviour of magnetorheological materials," Smart Mater. Struct. 5, 607-614 (1996).

${ }^{3}$ M. R. Jolly, J. W. Bender, and J. D. Carlson, "Properties and applications of commercial magnetorheological fluids," in 5th Annual International Symposium on Smart Structures and Materials (International Society for Optics and Photonics, 1998), pp. 262-275. 
${ }^{4}$ D. J. Klingenberg, "Magnetorheology: Applications and challenges," AIChE J. 47, 246-249 (2001).

${ }^{5}$ A.-G. Olabi and A. Grunwald, "Design and application of magnetorheological fluid," Mater. Des. 28, 2658-2664 (2007).

${ }^{6}$ J. Segovia-Gutiérrez, C. Berli, and J. De Vicente, "Nonlinear viscoelasticity and two-step yielding in magnetorheology: A colloidal gel approach to understand the effect of particle concentration," J. Rheol. 56, 1429-1448 (2012).

${ }^{7}$ G. Bossis, E. Lemaire, O. Volkova, and H. Clercx, "Yield stress in magnetorheological and electrorheological fluids: A comparison between microscopic and macroscopic structural models," J. Rheol. 41, 687-704 (1997).

${ }^{8}$ J. de Vicente, D. J. Klingenberg, and R. Hidalgo-Alvarez, "Magnetorheological fluids: A review," Soft Matter 7, 3701-3710 (2011).

${ }^{9}$ G. Bossis, P. Khuzir, S. Lacis, and O. Volkova, "Yield behavior of magnetorheological suspensions," J. Magn. Magn. Mater. 258-259, 456-458 (2003).

${ }^{10}$ R. Bonnecaze and J. Brady, "Yield stresses in electrorheological fluids," J. Rheol. 36, 73-115 (1992).

${ }^{11}$ M. Parthasarathy and D. J. Klingenberg, "A microstructural investigation of the nonlinear response of electrorheological suspensions: I. Start-up of steady shear," Rheol. Acta 34, 417-429 (1995).

${ }^{12}$ D. Kittipoomwong, D. J. Klingenberg, and J. C. Ulicny, "Dynamic yield stress enhancement in bidisperse magnetorheological fluids," J. Rheol. 49, 1521-1538 (2005).

${ }^{13}$ M. T. López-López, P. Kuzhir, J. Caballero-Hernandez, L. Rodríguez-Arco, J. D. Duran, and G. Bossis, "Yield stress in magnetorheological suspensions near the limit of maximum-packing fraction," J. Rheol. 56, 1209 (2012).

${ }^{14}$ J. Rodríguez-López, P. C. Blázquez, L. Elvira, F. M. de Espinosa, J. Ramírez, and J. de Vicente, "On the yielding behaviour in magnetorheology using ultrasounds, shear and normal stresses, and optical microscopy," J. Phys. D: Appl. Phys. 48, 465503 (2015).

${ }^{15}$ J. de Vicente, M. T. López-López, J. D. Durán, and F. González-Caballero, "Shear flow behavior of confined magnetorheological fluids at low magnetic field strengths," Rheol. Acta 44, 94-103 (2004).

${ }^{16}$ J. Ramos, D. Klingenberg, R. Hidalgo-Alvarez, and J. de Vicente, "Steady shear magnetorheology of inverse ferrofluids," J. Rheol. 55, 127-152 (2011).

${ }^{17}$ J. Ramos, J. de Vicente, and R. Hidalgo-Alvarez, "Small-amplitude oscillatory shear magnetorheology of inverse ferrofluids," Langmuir 26, 9334-9341 (2010).

${ }^{18}$ W. Li, H. Du, G. Chen, and S. H. Yeo, "Viscoelastic properties of MR fluids under oscillatory shear," Proc. SPIE 4331, 333-342 (2001).

${ }^{19}$ J. A. Ruiz-López, J. C. Fernández-Toledano, D. J. Klingenberg, R. HidalgoAlvarez, and J. de Vicente, "Model magnetorheology: A direct comparative study between theories, particle-level simulations and experiments, in steady and dynamic oscillatory shear," J. Rheol. 60, 61-74 (2016).

${ }^{20}$ W. H. Li, H. Du, G. Chen, S. H. Yeo, and N. Guo, "Nonlinear viscoelastic properties of MR fluids under large-amplitude-oscillatory-shear," Rheol. Acta 42, 280-286 (2003).

${ }^{21}$ K. Hyun, S. H. Kim, K. H. Ahn, and S. J. Lee, "Large amplitude oscillatory shear as a way to classify the complex fluids," J. Non-Newtonian Fluid Mech. 107, 51-65 (2002).

${ }^{22}$ J. E. Martin and J. Odinek, "A light-scattering study of the nonlinear dynamics of electrorheological fluids in oscillatory shear," J. Rheol. 39, 995-1009 (1995).

${ }^{23}$ H. G. Sim, K. H. Ahn, and S. J. Lee, "Three-dimensional dynamics simulation of electrorheological fluids under large amplitude oscillatory shear flow," J. Rheol. 47, 879-895 (2003).

${ }^{24}$ S. S. Deshmukh, "Development, characterization and applications of magnetorheological fluid based "smart' materials on the macro-to-micro scale," $\mathrm{Ph} . D$. thesis, Massachusetts Institute of Technology, 2006.

${ }^{25}$ S. Jamali, G. H. McKinley, and R. C. Armstrong, "Microstructural rearrangements and their rheological implications in a model thixotropic elasto-visco-plastic (TEVP) fluid," Phys. Rev. Lett. 118, 048003 (2017)

${ }^{26}$ D. Klingenberg, F. van Swol, and C. Zukoski, "Dynamic simulation of electrorheological suspensions," J. Chem. Phys. 91, 7888-7895 (1989).

${ }^{27}$ J. Fernández-Toledano, J. Ruiz-López, R. Hidalgo-Álvarez, and J. de Vicente, "Simulations of polydisperse magnetorheological fluids: A structural and kinetic investigation," J. Rheol. 59, 475-498 (2015).
${ }^{28} \mathrm{H}$. Ly, K. Ito, H. Banks, M. Jolly, and F. Reitich, "Dynamic simulation of the temporal response of microstructure formation in magnetorheological fluids," Int. J. Mod. Phys. B 15, 894-903 (2001).

${ }^{29}$ Y. Pappas and D. J. Klingenberg, "Simulations of magnetorheological suspensions in poiseuille flow," Rheol. Acta 45, 621-629 (2006).

${ }^{30}$ J. De Vicente, M. López-López, J. Durán, and G. Bossis, “A slender-body micromechanical model for viscoelasticity of magnetic colloids: Comparison with preliminary experimental data," J. Colloid Interface Sci. 282, 193-201 (2005).

${ }^{31}$ A. Gómez-Ramírez, P. Kuzhir, M. López-López, G. Bossis, A. Meunier, and J. Durán, "Steady shear flow of magnetic fiber suspensions: Theory and comparison with experiments," J. Rheol. 55, 43-67 (2011).

${ }^{32} \mathrm{D}$. Klingenberg, "Simulation of the dynamic oscillatory response of electrorheological suspensions: Demonstration of a relaxation mechanism," J. Rheol. 37, 199-214 (1993).

${ }^{33}$ M. Parthasarathy and D. J. Klingenberg, "Large amplitude oscillatory shear of ER suspensions," J. Non-Newtonian Fluid Mech. 81, 83-104 (1999).

${ }^{34}$ B.-J. de Gans, H. Hoekstra, and J. Mellema, "Non-linear magnetorheological behaviour of an inverse ferrofluid," Faraday Discuss. 112, 209-224 (1999).

${ }^{35}$ T. G. Kang, M. A. Hulsen, J. M. den Toonder, P. D. Anderson, and H. E. Meijer, "A direct simulation method for flows with suspended paramagnetic particles," J. Comput. Phys. 227, 4441-4458 (2008).

${ }^{36}$ M. R. Hashemi, M. T. Manzari, and R. Fatehi, "Direct numerical simulation of magnetic particles suspended in a Newtonian fluid exhibiting finite inertia under SAOS" (unpublished).

${ }^{37}$ T. G. Kang, M. A. Hulsen, and J. M. den Toonder, "Dynamics of magnetic chains in a shear flow under the influence of a uniform magnetic field," Phys. Fluids 24, 042001 (2012)

${ }^{38}$ M. R. Hashemi, M. T. Manzari, and R. Fatehi, "A SPH solver for simulating paramagnetic solid fluid interaction in the presence of an external magnetic field," Appl. Math. Modell. 40, 4341-4369 (2016).

${ }^{39}$ M. Wilhelm, "Fourier-transform rheology," Macromol. Mater. Eng. 287, 83-105 (2002).

${ }^{40}$ R. H. Ewoldt, "Nonlinear viscoelastic materials: Bioinspired applications and new characterization measures," Ph.D. thesis, Massachusetts Institute of Technology, 2009.

${ }^{41}$ M. Mermet-Guyennet, J. G. de Castro, M. Habibi, N. Martzel, M. Denn, and D. Bonn, "LAOS: The strain softening/strain hardening paradox," J. Rheol. 59, 21-32 (2015).

${ }^{42}$ M. R. Hashemi, M. T. Manzari, and R. Fatehi, "Evaluation of a pressure splitting formulation for weakly compressible SPH: Fluid flow around periodic array of cylinders," Comput. Math. Appl. 71, 758-778 (2016).

${ }^{43}$ J. A. Stratton, Electromagnetic Theory (John Wiley \& Sons, 1941).

${ }^{44}$ D. C. Jiles, Introduction to Magnetism and Magnetic Materials (CRC Press, 1998).

${ }^{45}$ S. Kang and Y. Suh, "An immersed-boundary finite-volume method for direct simulation of flows with suspended paramagnetic particles," Int. J. Numer. Methods Fluids 67, 58-73 (2011).

${ }^{46}$ D. J. Klingenberg, J. C. Ulicny, and M. A. Golden, "Mason numbers for magnetorheology," J. Rheol. 51, 883-893 (2007).

${ }^{47}$ M. J. Reimers and J. M. Dealy, "Sliding plate rheometer studies of concentrated polystyrene solutions: Large amplitude oscillatory shear of a very high molecular weight polymer in diethyl phthalate," J. Rheol. 40, 167-186 (1996).

${ }^{48}$ R. H. Ewoldt, A. Hosoi, and G. H. McKinley, "New measures for characterizing nonlinear viscoelasticity in large amplitude oscillatory shear," J. Rheol. 52, 1427-1458 (2008).

${ }^{49}$ R. Ewoldt, P. Winter, and G. McKinley, MITlaos version 2.1 Beta for MATLAB, Self-Published, Cambridge, MA, 2007.

${ }^{50}$ K. S. Cho, K. Hyun, K. H. Ahn, and S. J. Lee, "A geometrical interpretation of large amplitude oscillatory shear response," J. Rheol. 49, 747-758 (2005).

${ }^{51}$ K. Hyun, M. Wilhelm, C. O. Klein, K. S. Cho, J. G. Nam, K. H. Ahn, S. J. Lee, R. H. Ewoldt, and G. H. McKinley, "A review of nonlinear oscillatory shear tests: Analysis and application of large amplitude oscillatory shear (LAOS)," Prog. Polym. Sci. 36, 1697-1753 (2011).

${ }^{52}$ A. Fernandez-Nieves and A. M. Puertas, Fluids, Colloids and Soft Materials: An Introduction to Soft Matter Physics (Wiley Online Library, 2016), Vol. 7.

${ }^{53}$ R. H. Ewoldt and N. A. Bharadwaj, "Low-dimensional intrinsic material functions for nonlinear viscoelasticity," Rheol. Acta 52, 201-219 (2013). 
${ }^{54}$ W. R. Hwang, M. A. Hulsen, and H. E. Meijer, "Direct simulation of particle suspensions in sliding bi-periodic frames," J. Comput. Phys. 194, 742-772 (2004).

${ }^{55}$ N. Patankar, P. Huang, T. Ko, and D. Joseph, "Lift-off of a single particle in Newtonian and viscoelastic fluids by direct numerical simulation," J. Fluid Mech. 438, 67-100 (2001).
${ }^{56}$ F. Ding, A. J. Giacomin, R. B. Bird, and C.-B. Kweon, "Viscous dissipation with fluid inertia in oscillatory shear flow," J. Non-Newtonian Fluid Mech. 86, 359-374 (1999).

${ }^{57}$ H. M. Wyss, K. Miyazaki, J. Mattsson, Z. Hu, D. R. Reichman, and D. A. Weitz, "Strain-rate frequency superposition: A rheological probe of structural relaxation in soft materials," Phys. Rev. Lett. 98, 238303 (2007). 